\title{
A synthesis of European seahorse taxonomy, population structure, and habitat use as a basis for assessment, monitoring and conservation
}

\author{
Lucy C. Woodall ${ }^{1,2} \circledast$. Francisco Otero-Ferrer ${ }^{3} \cdot$ Miguel Correia $^{2,4} \cdot$ Janelle M. R. Curtis $^{5} \cdot$ Neil Garrick-Maidment $^{6}$. \\ Paul W. Shaw ${ }^{7} \cdot$ Heather J. Koldewey ${ }^{2,8}$
}

Received: 15 August 2017 / Accepted: 22 November 2017 / Published online: 5 December 2017

(C) The Author(s) 2017. This article is an open access publication

\begin{abstract}
Accurate taxonomy, population demography, and habitat descriptors inform species threat assessments and the design of effective conservation measures. Here we combine published studies with new genetic, morphological and habitat data that were collected from seahorse populations located along the European and North African coastlines to help inform management decisions for European seahorses. This study confirms the presence of only two native seahorse species (Hippocampus guttulatus and H. hippocampus) across Europe, with sporadic occurrence of non-native seahorse species in European waters. For the two native species, our findings demonstrate that highly variable morphological characteristics, such as size and presence or number of cirri, are unreliable for distinguishing species. Both species exhibit sex dimorphism with females being significantly larger. Across its range, $H$. guttulatus were larger and found at higher densities in cooler waters, and individuals in the Black Sea were significantly smaller than in other populations. H. hippocampus were significantly larger in Senegal. Hippocampus guttulatus tends to have higher density populations than H. hippocampus when they occur sympatrically. Although these species are often associated with seagrass beds, data show both species inhabit a wide variety of shallow habitats and use a mixture of holdfasts. We suggest an international mosaic of protected areas focused on multiple habitat types as the first step to successful assessment, monitoring and conservation management of these Data Deficient species.
\end{abstract}

Responsible Editor: K. D. Clements.

Reviewed by R. Calado and an undisclosed expert.

Electronic supplementary material The online version of this article (https://doi.org/10.1007/s00227-017-3274-y) contains supplementary material, which is available to authorized users.

Lucy C. Woodall

Lucy.woodall@zoo.ox.ac.uk

1 Department of Zoology, University of Oxford, Oxford, UK

2 Project Seahorse, Zoological Society of London, Regent's Park, London, UK

3 Grupo en Biodiversidad y Conservación, IU-ECOAQUA, Universidad de Las Palmas de Gran Canaria, Crta. Taliarte s/n, 35214 Telde, Spain

4 CCMar, Universidade do Algarve, F. C. T., Edificio 7, Campus de Gambelas, 8005-139 Faro, Portugal

\section{Introduction}

The paucity of species-specific data is among the many challenges to designing effective marine conservation measures that are resilient to the enduring threats of climate change, coastal development, over-fishing, by-catch effects and invasive species (Klein et al. 2013; Selig et al. 2014). These challenges are further compounded when the taxonomy of species is uncertain. Knowing which species occur and

5 Pacific Biological Station, Fisheries and Oceans Canada, 3190 Hammond Bay Road, Nanaimo, BC, Canada

6 The Seahorse Trust, 36 Greatwood Terrace, Topsham, Devon, UK

7 Institute of Biological, Environmental and Rural Sciences (IBERS), Aberystwyth University, Aberystwyth, UK

8 Centre for Ecology and Conservation, University of Exeter, Penryn, UK 
understanding their life-history, ecology, and behaviour is increasingly important to ensure effective and robust conservation and management (Perry et al. 2005; Lavergne et al. 2010; Dawson et al. 2011).

The cryptic nature of seahorses (genus Hippocampus) has led to significant confusion regarding their taxonomy and ecology, which poses challenges to managing the activities that threaten these fishes. The most recent and comprehensive taxonomic review suggests there are two native species of seahorse in European waters, $H$. guttulatus and $H$. hippocampus (Lourie et al. 2016), but considerable intraspecific variability in morphology within this genus (Lourie et al. 1999b; Otero-Ferrer et al. 2017) has led to much confusion regarding their taxonomy, and the taxonomy and nomenclature of these species is not stable. Authors previously suggested many additional species within this geographic range, based on small morphometric differences (Kuiter 2009). For instance a study by Vasil'Eva (2007), which has not been adopted (Eschmeyer and Fricke 2016), attempted to change the names of these species, while another author suggested additional species were present based on photographs (Kuiter 2009). There is ongoing discussion as to whether $H$. ramulosus is a simple synonym of $H$. guttulatus, and whether the regional morphological differences observed across the seahorse populations in the region are indicative of different species (Kuiter 2009). Taxonomic controversy involving splitting and lumping of species is common throughout the Syngnathidae family, due to limited discriminating morphological characteristics between species and the ability within the family to change colour and cirri (filamentous skin appendages) (Curtis 2006). As most ecological studies of seahorses in Europe have used the nomenclature of $H$. hippocampus and $H$. guttulatus to define their focal species (e.g. Curtis and Vincent 2005; Kitsos et al. 2008; Ben Amor et al. 2011; Caldwell and Vincent 2012; Filiz and Taskavak 2012; Gristina et al. 2015), there is some consensus for a conservative view of seahorse taxonomy. Some reports also suggest range extensions into European waters by non-native species: $H$. algiricus presence in the Canary Islands (Otero-Ferrer et al. 2015b, 2017), the Lessepsian migrant $H$. fuscus in the eastern Mediterranean (Golani and Fine 2002), and occasional rare migrants (e.g. H. erectus, Woodall et al. 2009). Therefore genetic data are particularly useful to clarify taxonomy and complement morphological data (Padial et al. 2010).

The two European seahorses H. guttulatus and H. hippocampus are the currently recognised names used in the IUCN Red List of Threatened Species (2012) and both are currently assessed as Data Deficient (Woodall 2012a, b). Both species have a large geographic range extending across most of Europe and North Africa including the Atlantic Ocean, Mediterranean and Black Seas (Lourie et al. 1999b; Otero-Ferrer et al. 2017). Neither species is thought to be currently targeted by fisheries throughout most of their geographic range, but there is trade in west Africa of H. hippocampus (Cisneros-Montemayor et al. 2016) and a new and increasing fishery for $\mathrm{H}$. guttulatus in the Ria Formosa in Portugal (M. Correia, pers. obs.). Both species are also susceptible to anthropogenic activities and habitat loss (Curtis et al. 2007). Ecological data on seahorses are scarce due to their apparent patchy distribution and low density, as well as their cryptic nature (Foster and Vincent 2004). These features make them particularly difficult to survey, assess and monitor the status of their populations, either for scientific research or commercial development projects, such as environmental impact assessments prior to construction work.

To date, a range-wide ecological assessment has been conducted for just one seahorse species (H. capensis), which is confined to three estuaries in South Africa (Lockyear et al. 2006). For European seahorses, research has generally been limited to small focal sites (e.g. Curtis and Vincent 2006; Gristina et al. 2015) or collection of qualitative data (e.g. Filiz and Taskavak 2012). However, a very large sighting dataset has been collected for UK and Ireland (N. GarrickMaidment pers. comm.). Comparisons of population structure among studies is also challenging because seahorse length can be measured by standard length $\left(L_{S}\right)$, total length $\left(L_{\mathrm{T}}\right)$ or height (Lourie et al. 1999a) and previous studies have used all of these (e.g. Verdiell-Cubedo et al. 2008; Nadeau et al. 2009; Caldwell and Vincent 2012; Vieira et al. 2014).

Focusing on the taxonomy, biology and life history of European seahorses, we use published and unpublished sources of genetic, demographic and environmental data to investigate the following objectives:

1. Use genetic markers to confirm the number of seahorse species present in Europe

2. Test for differences in population structure and behaviour throughout the range

3. Test for the correlation of population structure and morphology with environmental variables

This information will help to advance our ability to effectively manage Hippocampus spp. within Europe, by providing detailed information that can help determine appropriate protection and mitigation interventions as well as the accurate assessment of seahorse populations.

\section{Materials and methods}

\section{Geographic extent, literature sources and standardization}

The geographic extent of this review covers seahorse populations from the Northeast Atlantic Ocean, including the 
Macaronesian islands, and the Mediterranean and Black Seas. In total, data from 13 countries and 37 different sites are reviewed. These data cover the known geographic range of $H$. guttulatus and $H$. hippocampus (Lourie et al. 1999b), however most individual studies generally focused on sites in Portugal and the eastern Mediterranean due to these being identified as having relatively high seahorse abundance that led to longer term studies. Data used in this review were from a wide range of sources, including sources known to authors or found using a combination of the following search terms 'Seahorse, Hippocampus, guttulatus, ramulosus, Mediterranean, Atlantic, Black Sea, short snouted, long snouted, Hippocampe, Caballito de Mar, cavalos marinhos' in search engines Google Scholar and Web of Science. Sources include new data and published literature comprising peer-reviewed papers, theses, books and grey literature such as conference posters and reports. Due to the diversity of the methods employed by these studies, we were not able to use all data from all studies in comparisons among sites. However, all density measures were standardised to ind. $\mathrm{m}^{-2}$ and seahorse length measurements were compiled as standard length $\left(L_{\mathrm{S}}\right)$ (Curtis and Vincent 2006), height (Foster and Vincent 2004) or total length $\left(L_{\mathrm{T}}\right)$ (Verdiell-Cubedo et al. 2008).

\section{New sample acquisition and genetic analysis}

Most specimen tissue was collected during sampling dives. Further specimens or tissue and associated data were also donated by fishers, public aquariums and academics, and were used when source location was known. In total, seahorse tissue was obtained from specimens from 18 sites around Europe and North Africa (Fig. 1). Authors directly sampled tissues from 14 sites, while tissue from the remaining four locations was donated by other researchers. The mitochondrial DNA cytochrome b gene (cytb) and Control Region (CR) were amplified from specimens using methods given in Woodall et al. $(2011,2015)$. All DNA sequences were deposited in Genbank (Table S1). Cytb is most routinely sequenced in seahorses, and thus provided an opportunity to include the greatest number of species. The cytb sequences were combined with seahorse reference sequences (Casey et al. 2004; Teske et al. 2007a, b), and aligned using ClustalW (Larkin et al. 2007) implemented in Geneious v6.1.7. The pipefish Syngnathus temminckii was used as an outgroup. A phylogenetic tree, to group similar haplotypes, was created in Mr. Bayes (Huelsenbeck and Ronquist 2001) implemented in Geneious after using Find model (Posada and Crandall 2001) to determine the most suitable nucleotide substitution model $(\mathrm{GTR}+\gamma)$ for this dataset.

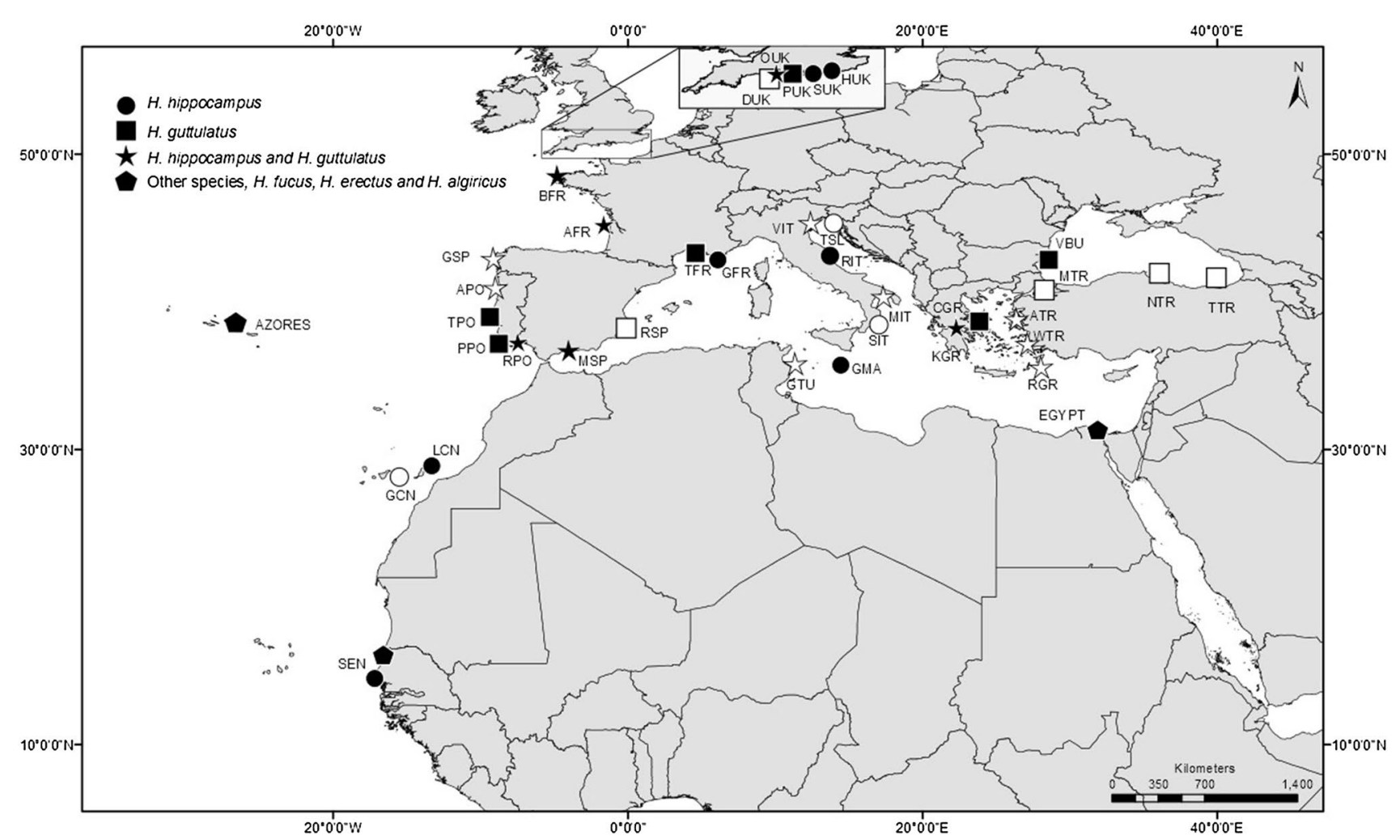

Fig. 1 Locations of seahorse tissue collection, population demography and environmental data, including site codes. Filled shapes are sites with new data and open shapes are sites with published data 


\section{Environmental and demographic data collection}

Environmental and demographic data were collected opportunistically during SCUBA dives, and morphological data from donated specimens as detailed below. Ideally, a stratified random or systematic sampling regime would be used to capture the full range of diversity in genetic and demographic structure and to identify environmental correlates. However, this was not feasible at the geographic scale of interest because so little information is known of seahorse distributions, which are generally patchy and low-density (Curtis and Vincent 2005). Instead local knowledge of seahorse occurrences was used to identify suitable and accessible survey locations. All seahorses encountered were identified in the field as either $H$. guttulatus or H. hippocampus, using morphological characteristics that have proved to be robust such as head shape and head:snout ratio (Lourie et al. 1999b; Curtis 2006). Photographs of specimens were taken when possible and representative images displayed in Fig. S1. Water temperature was extracted from http://www. seatemperature.org (1st July 2015) and was used for all sites where published studies were conducted.

\section{Survey data collected by divers}

At 14 of the 37 sites, diving methods were employed to sample seahorse populations in two ways: (a) to collect tissue samples for genetic studies (collection dives-see Woodall et al. 2011); or (b) to carry out rapid population assessments (transect dives-adapted from Curtis and Vincent 2005; Woodall et al. 2015). All dives were conducted during the main breeding season for the seahorses (May-October, Curtis and Vincent 2006). During both dive types (collection and transect), once individual seahorses were located, their holdfast (seagrass species, algae species, artificial structures, sand, shells, sessile invertebrates), depth and macro habitat (seagrass, macroalgae, sessile invertebrates, sponge, sand/ mud, stones/pebbles, rocks, cliff or artificial structures) were recorded, as well as species, sex, maturity (size when brood pouch is mature in males of that population; Curtis and Vincent 2006; Curtis et al. 2017), presence or absence of cirri (Curtis 2006) and straight trunk length $\left(L_{\mathrm{Tr}}\right)$ which was later converted to standard length $\left(L_{S}\right)$ (Curtis and Vincent 2006). Water clarity was assessed during each dive by estimating the horizontal visible distance (meters). The mean temperature at each site was calculated and recorded using a dive computer (Mosquito, Suunto).

\section{(a) Collection dives}

Between two and five (site dependant) divers searched the benthic substratum for seahorses using a random search pattern. The total time spent searching (diver hours) and number of individual seahorses located were recorded, but using this search pattern it was not possible to record the area of benthos searched. Search effort was measured by time and the number of searching divers, so the relative abundance of seahorses (seahorse per diver hour) could be reported (Schmitt and Sullivan 1996).

(b) Transect dives

A random position within the general search area was assigned as the starting point. This point was defined either by its GPS position or by its bearing and distance from a known structure (e.g. pier or rocky feature). From the starting position, a $30 \mathrm{~m}$ tape was laid out by one diver in a random direction while the other diver recorded the number of seahorses by species, sex and holdfast within a $2 \mathrm{~m}$ corridor belt transect centred along the tape length. Returning along the transect, both divers assessed the habitat by determining the dominant habitat type, which was defined by the broad categories given above. The divers also determined the percentage of cover of each habitat type within three randomly positioned $1 \mathrm{~m}^{2}$ quadrats. This process was repeated so that a total of four transects were surveyed per site. At one site in Greece (KGR - Fig. 1), the transects originated from a start line running at right angles to the slope. These transects were positioned to run parallel to the slope contours, at randomly assigned distances along the starting line. The deepest transect was surveyed first and the shallowest last. This method was necessary at KGR as it had a rapidly sloping benthic profile, which was absent from other sites.

\section{Commercial trade and fishing data}

Fisher data were collected from two locations in the UK. The seahorses were accidentally captured in gill nets and crab pots by local fishers who were targeting Solea solea and Cancer pagurus. Undamaged seahorses were returned to the water and injured ones were donated to local public aquariums. Environmental (habitat and depth) and seahorsespecific data (species and number) were recorded on the fishing boat and at the aquariums. Seahorses were donated by researchers from three locations in France, Portugal and Italy (AFR, TPO and RIT Table 1, Fig. 1). Seahorses from site AFR were collected during experimental trawls that were used to survey fish diversity in the bay. These seahorses were returned to their collection site following $L_{\mathrm{Tr}}$ measurement and photographing. Sites TPO and RIT were fished using beach seine nets by aquarium staff for specimen provision to local public aquariums. Specimens were measured and photographed by aquarium staff. Specimens from Senegal (SEN) were donated by Project Seahorse. Most of these Senegalese samples were obtained from a traditional medicine market (Hong Kong) by representatives of a Project 
Table 1 Sample sites of seahorses Hippocampus guttulatus (G) and H. hippocampus $(\mathrm{H})$, sampling method [Fishing Method (Net, Pot, Trawl, Trammel or Dredge), Type of dive (Collection, Survey or
Transect), Social (Donations, Interviews or Trade)], and environmental parameters (depth, visibility, temperature, habitat and main seahorse holdfast), (a) new data (b) published data

(a) New data

\begin{tabular}{|c|c|c|c|c|c|c|c|c|c|}
\hline Site & Country & Location type & Species & $\begin{array}{l}\text { Sampling } \\
\text { method }\end{array}$ & Depth range (m) & Visibility (m) & $\begin{array}{l}\text { Tem- } \\
\text { perature } \\
\left({ }^{\circ} \mathrm{C}\right)\end{array}$ & Holdfast & Habitat \\
\hline HUK & UK & Coast & $\mathrm{H}$ & Fisher-Nets & $\approx 55$ & $\mathrm{n} / \mathrm{a}$ & $\mathrm{n} / \mathrm{a}$ & Plocamium spp. & $\begin{array}{l}\text { Sand and mac- } \\
\text { roalgae }\end{array}$ \\
\hline SUK & UK & Coast & $\mathrm{H}$ & Fisher-Pots & $\approx 25$ & $\mathrm{n} / \mathrm{a}$ & $\mathrm{n} / \mathrm{a}$ & $\mathrm{n} / \mathrm{a}$ & Mussel bed \\
\hline BFR & France & Coastal & $\mathrm{H}, \mathrm{G}$ & Collection dive & $2-6$ & & 16 & $\begin{array}{l}\text { Z. marina, Ulva } \\
\text { spp., Sabel- } \\
\text { lidae spp. }\end{array}$ & Z. marina beds \\
\hline AFR & France & Lagoon & $\mathrm{G}$ & Donation & $5-10$ & $\mathrm{n} / \mathrm{a}$ & $\mathrm{n} / \mathrm{a}$ & $\mathrm{n} / \mathrm{a}$ & $\begin{array}{l}\text { Channel of sand } \\
\text { with Z. marina } \\
\text { beds on sides }\end{array}$ \\
\hline TPO & Portugal & Lagoon & G & $\begin{array}{l}\text { Donation, col- } \\
\text { lection dive }\end{array}$ & $3-4$ & $2-4$ & 19 & Z. marina & $\begin{array}{l}\text { Z. marina beds on } \\
\text { sand }\end{array}$ \\
\hline PPO & Portugal & Estuary & G & Collection dive & $1-3$ & $<1$ & 19 & Artificial & $\begin{array}{l}\text { Ropes and other } \\
\text { artificial struc- } \\
\text { tures on heavy } \\
\text { silt }\end{array}$ \\
\hline RPO & Portugal & Lagoon & $\mathrm{H}, \mathrm{G}$ & Collection dive & $1-6$ & $1-7$ & 20 & $\begin{array}{l}\text { Sand, Z. marina, } \\
\text { C. nodosa, } \\
\text { Artificial }\end{array}$ & $\begin{array}{l}\text { Sparse } Z \text {. marina, } \\
\text { sand urchins and } \\
\text { macro-algae, } \\
\text { tunicates and } \\
\text { artificial }\end{array}$ \\
\hline MSP & Spain & Coastal & $\mathrm{H}, \mathrm{G}$ & Collection dive & $6-8$ & $2-5$ & 19 & Z. marina & $\begin{array}{l}\text { Mixed sparse } \\
\text { seagrass beds }\end{array}$ \\
\hline TFR & France & Lagoon & G & Collection dive & $2-4$ & $1-4$ & 21 & Various & $\begin{array}{l}\text { Mixed and com- } \\
\text { plex }\end{array}$ \\
\hline GFR & France & Coastal & $\mathrm{H}$ & Collection dive & $4.5-6$ & $<1$ & 15 & On benthos & $\begin{array}{l}\text { Heavy silt and } \\
\text { tunicates }\end{array}$ \\
\hline GMA & Malta & Coastal & $\mathrm{H}$ & Collection dive & $9-20$ & $15-40$ & 18 & Z. marina & $\begin{array}{l}\text { Sand/seagrass bed, } \\
\quad+70 \mathrm{~m} \text { deep } \\
\text { wall@20 } \mathrm{m}\end{array}$ \\
\hline KGR & Greece & Coastal & $\mathrm{H}, \mathrm{G}$ & $\begin{array}{l}\text { Collection dive } \\
\text { transect dive }\end{array}$ & $5-19$ & $15-20$ & 24 & Z. marina & $\begin{array}{l}\text { Mixed seagrass on } \\
\text { slope }\end{array}$ \\
\hline CGR & Greece & Coastal & $\mathrm{G}$ & $\begin{array}{l}\text { Collection dive } \\
\text { transect dive }\end{array}$ & $2-5$ & $15-20$ & 26 & Stones & $\begin{array}{l}\text { Sponge, rock and } \\
\text { pebble wells }\end{array}$ \\
\hline VBU & Bulgaria & Coastal & G & $\begin{array}{l}\text { Collection dive } \\
\text { transect dive }\end{array}$ & $5-6.5$ & 1 & 25 & $\begin{array}{l}\text { In mixed algae, } \\
\text { Dictyopteris } \\
\text { and Chaeto- } \\
\text { morpha spp. }\end{array}$ & Ulva spp. \\
\hline $\mathrm{LCN}$ & Spain & Coastal & $\mathrm{H}$ & $\begin{array}{l}\text { Collection dive } \\
\text { transect dive }\end{array}$ & $6-21$ & $10-30$ & 21 & $\begin{array}{l}\text { Artificial sub- } \\
\text { strates }\end{array}$ & $\begin{array}{l}\text { Rock, rope and } \\
\text { ship wreck }\end{array}$ \\
\hline SEN & Senegal & $\mathrm{n} / \mathrm{a}$ & $\mathrm{H}$ & Trade & $\mathrm{n} / \mathrm{a}$ & $\mathrm{n} / \mathrm{a}$ & $\mathrm{n} / \mathrm{a}$ & $\mathrm{n} / \mathrm{a}$ & $\mathrm{n} / \mathrm{a}$ \\
\hline
\end{tabular}

(b) Published data

\begin{tabular}{|c|c|c|c|c|c|c|c|c|}
\hline Site & Country & Type & Species & Sampling method & Depth range $(\mathrm{m})$ & Holdfast & Habitat & References \\
\hline DUK & UK & Coastal & G & Survey dive & $1-3$ & Z. marina & Z. marina & $\begin{array}{l}\text { Garrick-Maidment } \\
\text { et al. (2010) }\end{array}$ \\
\hline OUK & UK & Lagoon & $\mathrm{H}, \mathrm{G}$ & Interview & $0-17$ & $\begin{array}{l}\text { Algae and seagrass } \\
\text { (G) n/a (H) }\end{array}$ & $\begin{array}{l}\text { Algae }(H+G), \\
\text { sand }(H+G), \\
\text { mixed seagrass } \\
(G), \text { Oyster bed } \\
(H+G)\end{array}$ & $\begin{array}{l}\text { N. Garrick-Maidment } \\
\text { Pers. Corr. }\end{array}$ \\
\hline
\end{tabular}


Table 1 (continued)

(b) Published data

\begin{tabular}{|c|c|c|c|c|c|c|c|c|}
\hline Site & Country & Type & Species & Sampling method & Depth range $(\mathrm{m})$ & Holdfast & Habitat & References \\
\hline AFR & France & Lagoon & $\mathrm{H}, \mathrm{G}$ & Interview & $3-20$ & $\mathrm{n} / \mathrm{a}$ & $\begin{array}{l}\text { Z. marina, } Z \text { nolti, } \\
\text { sand, shells }\end{array}$ & Grima (2011) \\
\hline GSP & Spain & Coastal & $\mathrm{H}, \mathrm{G}$ & Survey dive & $2.5-8$ & $\begin{array}{l}\text { Macroalgae (G) } \\
\text { Seagrass }(H)\end{array}$ & $\begin{array}{l}\text { Sand }(G), \text { seagrass } \\
\text { macroalgae }\end{array}$ & $\begin{array}{l}\text { Valladares et al. } \\
\text { (2011), (2013) }\end{array}$ \\
\hline APO & Portugal & Estuary & $\mathrm{H}, \mathrm{G}$ & Net & $\mathrm{n} / \mathrm{a}$ & $\mathrm{n} / \mathrm{a}$ & $\mathrm{n} / \mathrm{a}$ & Veiga et al. (2009) \\
\hline RPO & Portugal & Lagoon & $\mathrm{H}, \mathrm{G}$ & $\begin{array}{l}\text { Transect dive and } \\
\text { net }\end{array}$ & $0-7$ & $\begin{array}{l}\text { Tunicates and shells } \\
(\mathrm{H}+\mathrm{G}) \text {, Artificial } \\
(\mathrm{G}), \text { sessile inver- } \\
\text { tebrates }(\mathrm{H}+\mathrm{G}), \\
\text { macroalgae } \\
(\mathrm{H}+\mathrm{G}) \text {, seagrass } \\
(\mathrm{H}+\mathrm{G})\end{array}$ & $\begin{array}{l}\text { Mixed seagrass and } \\
\text { macroalgae }(G), \\
\text { sessile inverte- } \\
\text { brates }(H+G), \\
\text { sand }(H)\end{array}$ & $\begin{array}{l}\text { Curtis and Vincent } \\
\text { (2005, 2006), Curtis } \\
\text { (2004), Caldwell } \\
\text { and Vincent (2012), } \\
\text { Correia et al. } \\
\text { (2015), Vieira et al. } \\
\text { (2014) }\end{array}$ \\
\hline RSP & Spain & Lagoon & G & Net & $2-3$ & $\mathrm{n} / \mathrm{a}$ & $\begin{array}{l}\text { C. nodosa, invasive } \\
\text { Caulerpa }\end{array}$ & $\begin{array}{l}\text { Verdiell-Cubedo } \\
\text { et al. (2006) }\end{array}$ \\
\hline TFR & France & Lagoon & G & Transect dive & $0-9$ & Artificial & $\begin{array}{l}\text { Sand, algae and } \\
\text { sparse seagrass }\end{array}$ & Louisy (2011) \\
\hline SIT & Italy & Coastal & $\mathrm{H}$ & Transect dive & $\mathrm{n} / \mathrm{a}$ & $\mathrm{n} / \mathrm{a}$ & Sand & Canese et al. (2007) \\
\hline MIT & Italy & Lagoon & $\mathrm{H}, \mathrm{G}$ & Transect dive & $0-512$ & Artificial $(H+G)$ & $\begin{array}{l}\text { Mixed sand, sparse } \\
\text { seagrass, dense } \\
\text { Ulva sp. }\end{array}$ & $\begin{array}{l}\text { Tiralongo and Balda- } \\
\text { cconi (2014), Gris- } \\
\text { tina et al. (2015) }\end{array}$ \\
\hline VIT & Italy & Lagoon & $\mathrm{H}, \mathrm{G}$ & Net & $\mathrm{n} / \mathrm{a}$ & $\mathrm{n} / \mathrm{a}$ & $\begin{array}{l}\text { Seagrass }(H+G) \\
\text { saltmarsh }(G)\end{array}$ & Franco et al. (2006) \\
\hline TSL & Slovenia & Coastal & G & Transect dive & $4-10$ & C. nodosa & C. nodosa & $\begin{array}{l}\text { Bonaca and Lipej } \\
\text { (2005) }\end{array}$ \\
\hline KGR & Greece & Coastal & G & Transect dive & $\mathrm{n} / \mathrm{a}$ & $\mathrm{n} / \mathrm{a}$ & $\mathrm{n} / \mathrm{a}$ & $\begin{array}{l}\text { Issaris and Katsane- } \\
\text { vakis (2010) }\end{array}$ \\
\hline MTR & Turkey & Coastal & G & Net & $0-2$ & $\mathrm{n} / \mathrm{a}$ & Seagrass, sand & Keskin (2007) \\
\hline TTR & Turkey & Coastal & G & Interview & $\mathrm{n} / \mathrm{a}$ & $\mathrm{n} / \mathrm{a}$ & $\mathrm{n} / \mathrm{a}$ & $\begin{array}{l}\text { Kasapoglu and Duz- } \\
\text { gunes (2014) }\end{array}$ \\
\hline NTR & Turkey & Coastal & G & Interview & $\mathrm{n} / \mathrm{a}$ & $\mathrm{n} / \mathrm{a}$ & $\mathrm{n} / \mathrm{a}$ & Başusta et al. (2014) \\
\hline WTR & Turkey & Coastal & $\mathrm{H}, \mathrm{G}$ & Interview & $0-30$ & $\mathrm{n} / \mathrm{a}$ & $\begin{array}{l}\text { Seagrass, rock, } \\
\text { mud, sand }\end{array}$ & $\begin{array}{l}\text { Filiz and Taskavak } \\
\text { (2012) }\end{array}$ \\
\hline ATR & Turkey & Coastal & $\mathrm{H}, \mathrm{G}$ & Interview and trawl & $\mathrm{n} / \mathrm{a}$ & $\mathrm{n} / \mathrm{a}$ & $\mathrm{n} / \mathrm{a}$ & $\begin{array}{l}\text { Gurkan and Taskavak } \\
\text { (2007) }\end{array}$ \\
\hline RGR & Greece & Coastal & $\mathrm{H}, \mathrm{G}$ & Trawl & $12-15$ & $\mathrm{n} / \mathrm{a}$ & seagrass & Kitsos et al. (2008) \\
\hline GTU & Tunisia & Lagoons & $\mathrm{H}, \mathrm{G}$ & $\begin{array}{l}\text { Trammel net and } \\
\text { dredge gear }\end{array}$ & $\mathrm{n} / \mathrm{a}$ & $\mathrm{n} / \mathrm{a}$ & $\mathrm{n} / \mathrm{a}$ & $\begin{array}{l}\text { Ben Amor et al. } \\
\text { (2011) }\end{array}$ \\
\hline GCN & Spain & Coastal & $\mathrm{H}$ & Transect dive & 15 & $\begin{array}{l}\text { Macroalgae, sessile } \\
\text { invertebrates, } \\
\text { seagrass }\end{array}$ & $\begin{array}{l}\text { Rock, sand, } C \text {. } \\
\text { nodosa, macroal- } \\
\text { gae, artificial }\end{array}$ & Otero-Ferrer (2011) \\
\hline
\end{tabular}

Seahorse/TRAFFIC partnership, but twelve were obtained by K. West directly from Senegal traders (West 2012).

\section{Statistical analysis}

Pearson correlation was used to assess all correlation relationships between seahorse size, sex and cirri presence. Differences in seahorse length, once juvenile data (Curtis and Vincent 2005) were removed, were assessed using GLM to determine whether there was a difference between sexes and between sites (when mature seahorse $n>10$ ), or a combination of the two. A post hoc Tukey pairwise comparison was used to determine at which sites seahorse lengths were significantly different. Differences in seahorse abundance were determined by Mann-Whitney test, and correlation between abundance of the two species was assessed with Pearson correlation. Deviation of sex ratios from equal was measured with Chi squared goodness of fit, and post hoc multiple test Benjamini-Hochberg correction. Correlation between abiotic parameters and species abundance was assessed with Spearman Rho. All these tests were implemented in Minitab v 17. Association of species presence 
with specific habitat parameters was calculated by ANOSIM in Primer v7.

\section{Results}

\section{Genetic differentiation}

In total, 478 seahorse specimens from 18 sites representing 10 countries (Fig. 1, Table 1) were PCR amplified and sequenced for both cytb and CR regions, with fragments trimmed to 518 and $397 \mathrm{bp}$, respectively to assist alignment. Data suggested the presence of a single specimen of $H$. erectus from the Azores (Woodall et al. 2009), three specimens of $H$. fuscus from Egypt, and five specimens of H. algiricus from Senegal (cytb data only shown, Fig. 2). All other specimens clearly group into two monophyletic clades corresponding to the two recognised European species H. guttulatus (212) or H. hippocampus (257). Intraspecific DNA sequence variation across all samples of $H$. guttulatus and H. hippocampus was low (1.23\% cytb and $1.49 \% \mathrm{CR}$, and $1.94 \%$ cytb and $1.96 \% \mathrm{CR}$, respectively), and identical (H. guttulatus) or similar (H. hippocampus) to variation observed within individual populations [maximum of $1.23 \%$ for cytb (VBU) and $1.49 \%$ for CR (MSP) in H. guttulatus, and $1.21 \%$ for cytb (RPO) and $1.67 \%$ for CR (SEN) in H. hippocampus-see Woodall et al. $(2011,2015)]$.

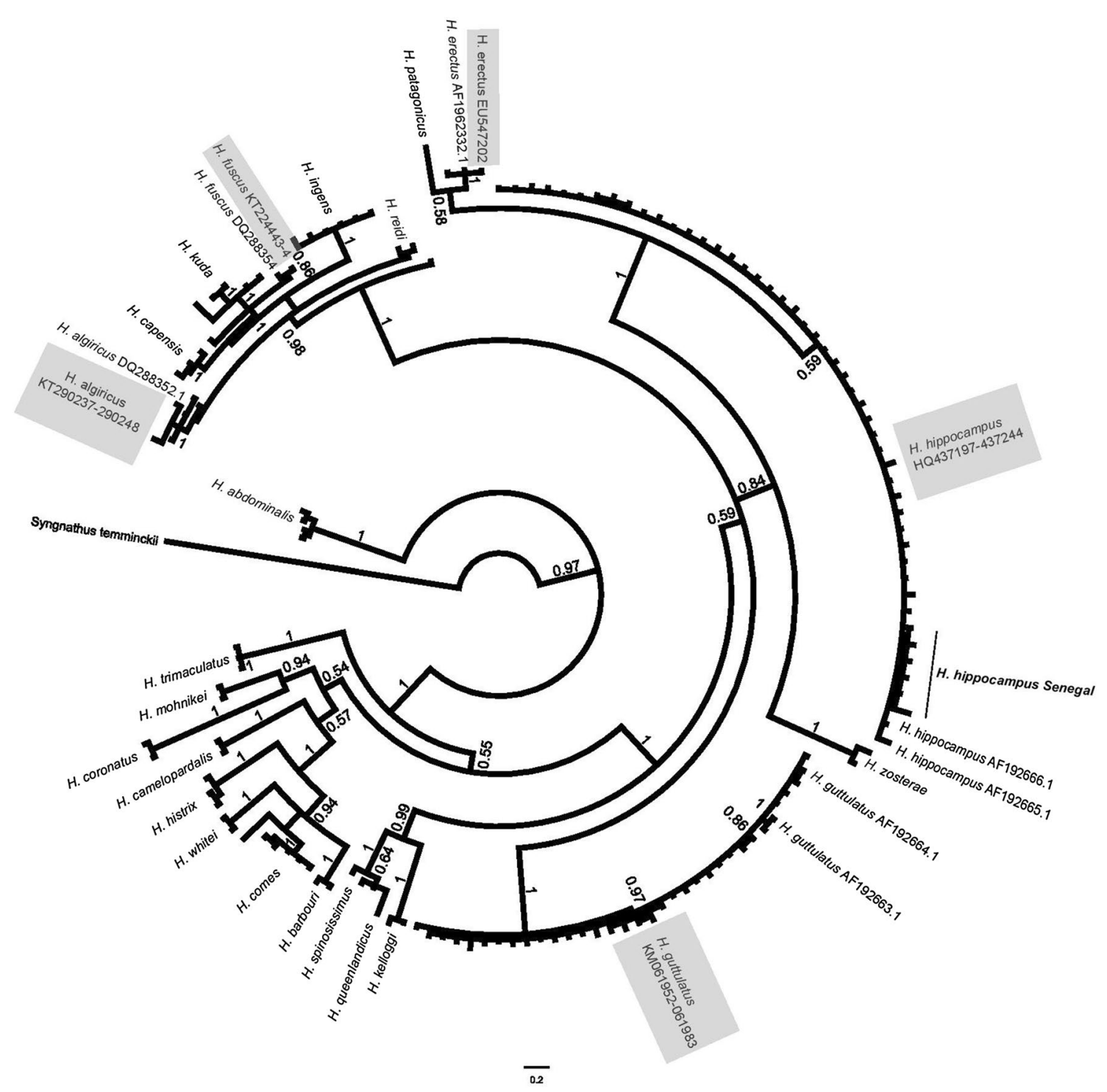

Fig. 2 Phylogenetic tree of the relationship among Hippocampus species, constructed from Cytochrome b using MrBayes (GTR $+\gamma)$ and shows posterior probability. Shaded labels are those generated in this study, and $H$. hippocampus from Senegal are denoted by bold text 


\section{Seahorse morphology}

Cirri were present on both species and the number of cirri present on seahorses varied considerably between sites and between individuals within sites. The mean cirri presence for $H$. guttulatus was $87 \%(40-100 \%, N=500)$ across 11 sites and for $H$. hippocampus the mean was $43 \%$ (6-74\%, $N=226$ ) across 9 sites, covering the entire geographic range of these species. For both species the number of cirri varied more between individuals within the same site than between species; however the species differed significantly in number of cirri (Mann-Whitney, $W=157, N_{1}=11, N_{2}=98$, $P<0.005)$. In H. guttulatus, data from this study show there is no correlation between sex and cirri presence (Pearson correlation, $R=-0.11, N=151, P=0.16$ ), however standard length and cirri presence are significantly correlated with larger fish having cirri more often than smaller fish (Pearson correlation, $R=0.40, N=151, P<0.001)$. By contrast female $H$. hippocampus were more often seen with cirri than males (Pearson correlation, $R=-0.27, N=91, P<0.01$ ).

Standard length of $H$. guttulatus was significantly different between sexes and between sites (GLM, sex $F=39.4$, $P<0.001$, sites $F=47.7, P<0.001$, site and sex $F=0.47$, $P=0.83$ ). Males were significantly smaller than females, and $H$. guttulatus in the Black Sea were significantly smaller than at any other site according to Tukey's pairwise comparisons at 95\% CI. When new and published data are combined, individuals of $H$. guttulatus in the Black Sea (VBU and TTR, Fig. 1) are smaller than specimens sampled from everywhere else (Fig. 3, Table 2). In H. hippocampus there were significant differences in standard length between sexes, sites and the interaction of the two, accounting for $55 \%$ of the variation seen (GLM, sex $F=50.8, P<0.001$, sites $F=4.9 P<0.005$, sex and site $F=8.6, P<0.001$ ). Similar to $H$. guttulatus, male $H$. hippocampus were significantly smaller than the females according to Tukey's pairwise comparisons at 95\% CI, and individuals from Senegal were significantly larger than those of all other sites (Fig. 3, Table 2). In both species there is no correlation between latitude and standard length (H. guttulatus Pearson correlation, $R=0.23, N=15, P=0.42 ; H$. hippocampus $R=-0.362$, $N=16, P=0.17$ ).

\section{Seahorse population density}

The total number of seahorses observed at each site varied considerably depending on species and survey method. The mean abundance of $H$. guttulatus was $3.15 \pm 1.08$ (mean $\pm \mathrm{SE}$ ) seahorses per diver hour for collection dives, and in transect surveys density was $0.076 \pm 0.06$ ind. $\mathrm{m}^{-2}$. For $H$. hippocampus, the mean abundance was 2.49 seahorses per diver hour but only one transect was conducted for this species, so no mean density is reported. When data from published studies were combined with transect dives in this study, mean abundance was greater in $H$. guttulatus than H. hippocampus (H. guttulatus: $0.04 \pm 0.01$ ind. $\mathrm{m}^{-2}$ $N=12 ;$ H. hippocampus: $0.003 \pm 0.001$ ind. $\mathrm{m}^{-2}, N=7$ ) (Table 3).

In all locations $(N=6)$ where the two species occurred sympatrically, the density of $H$. guttulatus was greater than that of H. hippocampus and in all but one case this was by at least an order of magnitude greater (Table 3). In the Ria
Fig. 3 Standard length of $H$. guttulatus (squares) and $H$. hippocampus (circles) including new data and that from published studies, when $n>10$. Filled in shapes are new data and open shapes are data from previous studies (Table 1 for site code details). Sites are grouped by region and are ordered from north to south or west to east depending on location

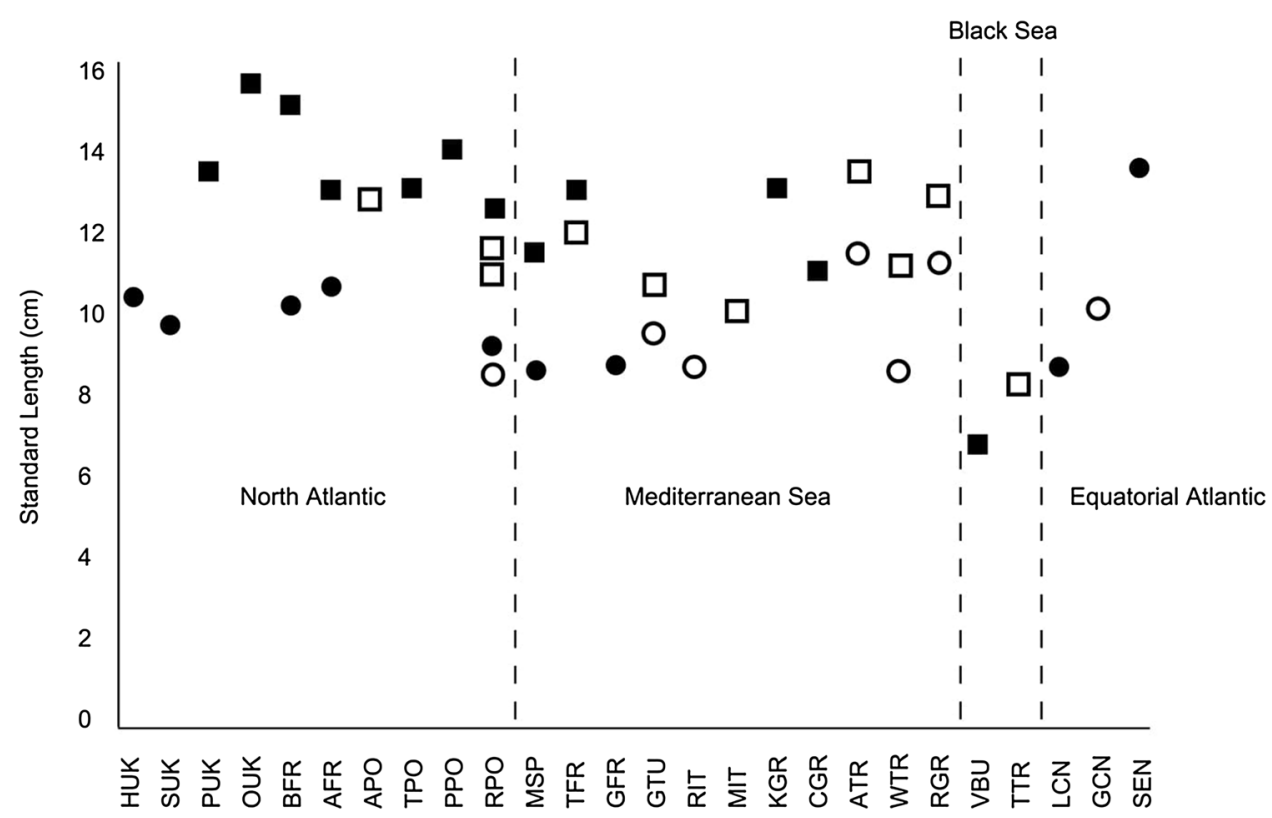

Sample site 
Table 2 Population demographics of Hippocampus guttulatus (a) and H. hippocampus (b)

\begin{tabular}{|c|c|c|c|c|c|c|}
\hline Site & Total number & Juvenile (\%) & $\begin{array}{l}\text { Proportion of } \\
\text { females }\end{array}$ & $\begin{array}{l}L_{\mathrm{s}}(\mathrm{cm}) \\
(\min \text { and } \max )\end{array}$ & Sampling period & References \\
\hline \multicolumn{7}{|c|}{ (a) Hippocampus guttulatus } \\
\hline PUK & 17 & 0 & 0.53 & $13.6(8.6-18.6)$ & May, Aug, Oct & This study \\
\hline OUK & 28 & $\mathrm{n} / \mathrm{a}$ & $\mathrm{n} / \mathrm{a}$ & $15.7(10.0-21.6)^{\mathrm{b}}$ & $\mathrm{n} / \mathrm{a}$ & Neil Garrick-Maidment Pers. Corr. \\
\hline BFR & 15 & 1 & 0.60 & $15.2(8.0-20.5)$ & June & This study \\
\hline AFR & 38 & 3 & 0.71 & $13.0(8.6-17.4)$ & Sept, Oct, Nov & This study \\
\hline GSP & 21 & 14 & 0.33 & $\mathrm{n} / \mathrm{a}$ & Year-round & Valladares et al. (2011) \\
\hline APO & 84 & $\mathrm{n} / \mathrm{a}$ & $\mathrm{n} / \mathrm{a}$ & $12.8(3.6-18.5)$ & Year-round & Veiga et al. (2009) \\
\hline TPO & 37 & 8 & 0.50 & $13.2(9.0-20.4)$ & Sept, Oct & This study \\
\hline PPO & 42 & 43 & 0.45 & $13.9(9.0-16.8)$ & Sept & This study \\
\hline \multirow[t]{5}{*}{ RPO } & 321 & 17 & 0.57 & $12.7(8.7-17.9)$ & Sept & This study \\
\hline & 384 & 13 & 0.55 & $11.3^{\mathrm{a}}(6.9-21.5)$ & May-Oct over 3 years & Curtis and Vincent (2006) \\
\hline & 58 & 10 & 0.57 & $\mathrm{n} / \mathrm{a}$ & July-Nov & Caldwell and Vincent (2012) \\
\hline & 1674 & 6 & 0.53 & $\mathrm{n} / \mathrm{a}$ & Year-round & Correia (2015) \\
\hline & 2042 & $\mathrm{n} / \mathrm{a}$ & $\mathrm{n} / \mathrm{a}$ & $11.7(7.1-16.6)^{\mathrm{b}}$ & Year-round & Vieira et al. (2014) \\
\hline MSP & 19 & 0 & 0.62 & $11.8(9.2-17.9)$ & June & This study \\
\hline RSP & 31 & $\mathrm{n} / \mathrm{a}$ & $\mathrm{n} / \mathrm{a}$ & $\mathrm{n} / \mathrm{a}(4.2-7.3)^{\mathrm{c}}$ & Year-round & Verdiell-Cubedo et al. (2006) \\
\hline \multirow[t]{2}{*}{ TFR } & 25 & 0 & 0.36 & $13.0(9.9-18.6)$ & June, July, Aug & This study \\
\hline & 114 & 16 & 0.62 & $12.0(8.1-16)^{\mathrm{b}}$ & Year-round & Louisy (2011) \\
\hline MIT & 225 & 21 & 0.54 & $10.0(7.0-14.0)$ & June-Sept & Gristina et al. (2015) \\
\hline KGR & 14 & 7 & 0.46 & $13.0(8.0-15.7)$ & Sept & This study \\
\hline CGR & 13 & 0 & 0.53 & $11.2(8.6-15.3)$ & Sept & This study \\
\hline VBU & 60 & 2 & 0.68 & $6.4(4.3-9.0)$ & June & This study \\
\hline TTR & 272 & $\mathrm{n} / \mathrm{a}$ & 0.50 & $8.3(6.5-10.3)^{\mathrm{c}}$ & Year-round & Kasapoglu and Duzgunes (2014) \\
\hline NTR & 139 & $\mathrm{n} / \mathrm{a}$ & 0.42 & $\mathrm{n} / \mathrm{a}(5.7-9.0)$ & $\mathrm{n} / \mathrm{a}$ & Başusta et al. (2014) \\
\hline WTR & 135 & $\mathrm{n} / \mathrm{a}$ & $\mathrm{n} / \mathrm{a}$ & $10.8(6.4-13.2)^{\mathrm{cd}}$ & $\mathrm{n} / \mathrm{a}$ & Filiz and Taskavak (2012) \\
\hline ATR & 200 & $\mathrm{n} / \mathrm{a}$ & 0.48 & $13.3(10.0-16.5)$ & Year-round & Gurkan and Taskavak (2007) \\
\hline RGR & 279 & $\mathrm{n} / \mathrm{a}$ & 0.54 & $10.8(7.8-22.5)$ & Mar & Kitsos et al. (2008) \\
\hline GTU & 1773 & $\mathrm{n} / \mathrm{a}$ & $\mathrm{n} / \mathrm{a}$ & $12.5(6.3-17.6)^{\mathrm{ce}}$ & Year-round & Ben Amor et al. (2011) \\
\hline \multicolumn{7}{|c|}{ (b) H. hippocampus } \\
\hline HUK & 49 & 40 & 0.61 & $10.5(5.6-19.8)$ & Sept & This study \\
\hline SUK & 24 & 33 & 0.50 & $9.9(7.1-16.8)$ & April & This study \\
\hline OUK & 9 & $\mathrm{n} / \mathrm{a}$ & $\mathrm{n} / \mathrm{a}$ & $9.4(5.1-15.2)$ & $\mathrm{n} / \mathrm{a}$ & Neil Garrick-Maidment Pers. Corr. \\
\hline BFR & 16 & 0 & 0.50 & $10.2(7.3-13.5)$ & June & This study \\
\hline AFR & 13 & 13 & 0.54 & $10.6(5.7-15.7)$ & Sept, Oct, Nov & This study \\
\hline GSP & 9 & $\mathrm{n} / \mathrm{a}$ & 0.34 & n/a $(11.8-17.1)$ & Year-round & Valladares et al. (2013) \\
\hline APO & 9 & $\mathrm{n} / \mathrm{a}$ & $\mathrm{n} / \mathrm{a}$ & $\mathrm{n} / \mathrm{a}(4.5-13.7)^{\mathrm{c}}$ & Year-round & Veiga et al. (2009) \\
\hline $\mathrm{PPO}$ & 6 & 0 & 0.33 & $8.3(4.3-14.9)$ & Sept & This study \\
\hline \multirow[t]{5}{*}{ RPO } & 44 & 0 & 0.60 & $8.7(4.3-17.6)$ & Sept, October & This study \\
\hline & 41 & 2 & 0.44 & n/a $(8.7-14.6)$ & June-Sept & Curtis and Vincent (2005) \\
\hline & 18 & 28 & 0.38 & $\mathrm{n} / \mathrm{a}$ & July-Nov & Caldwell and Vincent (2012) \\
\hline & 418 & $\mathrm{n} / \mathrm{a}$ & $\mathrm{n} / \mathrm{a}$ & $8.3(5.0-13.4)^{b}$ & Sept & Vieira et al. (2014) \\
\hline & 86 & 22 & 0.53 & $\mathrm{n} / \mathrm{a}$ & Sept & Correia (2015) \\
\hline MSP & 23 & 0 & 0.52 & $8.5(5.1-13.4)$ & May, June & This study \\
\hline GFR & 21 & 0 & 0.61 & $8.5(5.6-13.3)$ & July & This study \\
\hline GMA & 5 & 0 & 0.60 & $9.1(6.4-13.4)$ & Aug & This study \\
\hline MIT & 16 & 6 & $\mathrm{n} / \mathrm{a}$ & $\mathrm{n} / \mathrm{a}$ & June-Sept & Gristina et al. (2015) \\
\hline RIT & 46 & $\mathrm{n} / \mathrm{a}$ & $\mathrm{n} / \mathrm{a}$ & $8.4(5.7-10.3)$ & March & This study \\
\hline KGR & 8 & 0 & 0.50 & $7.9(5.7-11.1)$ & Sept & This study \\
\hline WTR & 279 & $\mathrm{n} / \mathrm{a}$ & $\mathrm{n} / \mathrm{a}$ & $8.4(5.2-12.8)^{\mathrm{d}}$ & $\mathrm{n} / \mathrm{a}$ & Filiz and Tasavak (2012) \\
\hline
\end{tabular}


Table 2 (continued)

\begin{tabular}{lclllll}
\hline Site & Total number & Juvenile $(\%)$ & $\begin{array}{l}\text { Proportion of } \\
\text { females }\end{array}$ & $\begin{array}{l}L_{\mathrm{s}}(\mathrm{cm}) \\
(\mathrm{min} \text { and max })\end{array}$ & Sampling period & References \\
\hline ATR & 29 & $\mathrm{n} / \mathrm{a}$ & 0.27 & $11.3(7.9-14.0)$ & Year-round & Gurkan and Taskavak (2007) \\
RGR & 19 & $\mathrm{n} / \mathrm{a}$ & 0.26 & $9.3(6.9-10.4)$ & Mar & Kitsos et al. (2008) \\
GTU & 236 & $\mathrm{n} / \mathrm{a}$ & $\mathrm{n} / \mathrm{a}$ & $10.9(7.4-15.6)$ & Year-round & Ben Amor et al. (2011) \\
LCN & 19 & 0 & 0.52 & $8.4(5.6-11.9)$ & Nov & This study \\
GCN & 165 & 20 & 0.58 & $10.2(7.7-14.7)$ & Year-round & Otero-Ferrer et al. (2015a) \\
SEN & 40 & n/a & n/a & $13.7(10.8-18.1)$ & n/a & This study \\
\hline
\end{tabular}

Number of seahorses samples, percentage of juveniles, sex ratio, standard length $\left(L_{\mathrm{s}}\right)$ and sampling period

${ }^{a}$ At first reproduction

${ }^{\mathrm{b}}$ Height

${ }^{\mathrm{c}}$ Total length

${ }^{\mathrm{d}}$ Dried specimens

${ }^{\mathrm{e}} \mathrm{ID}$ as H. ramulosus

Table 3 Mean population abundance include new and published data new for $H$. guttulatus and H. hippocampus

\begin{tabular}{|c|c|c|c|c|c|}
\hline \multirow[t]{2}{*}{ Site } & \multicolumn{2}{|c|}{ Seahorses per diver hour } & \multicolumn{2}{|c|}{ Seahorses per $\mathrm{m}^{2}$ of transect } & \multirow[t]{2}{*}{ References } \\
\hline & H. guttulatus & H. hippocampus & H. guttulatus & H. hippocampus & \\
\hline BFR & 1.565 & 1.130 & - & - & This study \\
\hline GSP & - & - & 0.007 & - & Valladares et al. (2011) \\
\hline TPO & 3.000 & 0.006 & - & - & This study \\
\hline PPO & 6.000 & 0.980 & - & - & This study \\
\hline \multirow[t]{3}{*}{ RPO } & - & - & 0.073 & 0.007 & Curtis and Vincent (2005) \\
\hline & - & - & 0.004 & 0.001 & Caldwell and Vincent (2012) \\
\hline & - & - & 0.107 & 0.005 & Correia (2015) \\
\hline \multirow[t]{2}{*}{ SFR } & 0.980 & $0.001^{\mathrm{a}}$ & - & - & This study \\
\hline & - & - & $0.001-0.014^{\mathrm{b}}$ & - & Louisy (2011) \\
\hline GFR & - & 7.000 & - & - & This study \\
\hline GMA & - & 0.190 & - & & This study \\
\hline SIT & - & - & - & 0.006 & Canese et al. (2007) \\
\hline MIT & - & - & 0.018 & $>0.001$ & Gristina et al. (2015) \\
\hline VIT & - & - & 0.001 & $>0.001$ & Franco et al. (2006) \\
\hline TSL & - & - & $0-0.08$ & - & Bonaca and Lipej (2005) \\
\hline \multirow[t]{2}{*}{ KGR } & 1.070 & 1.000 & 0.020 & 0 & This study \\
\hline & - & & 0.004 & - & Issaris and Katsanevakis (2010) \\
\hline CGR & 1.220 & 0.002 & 0.004 & 0 & This study \\
\hline VBU & $8.240^{\mathrm{c}}$ & - & 0.203 & - & This study \\
\hline $\mathrm{LCN}$ & - & 2.100 & - & 0.002 & This study \\
\hline GCN & - & $1.760 / 0.840^{\mathrm{d}}$ & - & - & Otero-Ferrer et al. (2015b) \\
\hline
\end{tabular}

${ }^{a}$ Only one seahorse seen but first report of this species here

${ }^{\mathrm{b}}$ Range given not mean abundance

${ }^{\mathrm{c}}$ Abundance estimate was limited by underwater genetic sampling procedures

${ }^{\mathrm{d}}$ Calculated from 15 min dive transects
Formosa, Portugal, where observations covered many years, $H$. guttulatus was always found in greater abundance than $H$. hippocampus when the Ria Formosa was considered as one site, but in some locations within the Ria Formosa only one of the two species was found (e.g. Curtis and Vincent 2005).
There is no significant difference in abundance of $H$. guttulatus or $H$. hippocampus between sites where it cohabits or not with its congener (H. guttulatus new data Mann-Whitney, $W=18, N=10, P=0.8 ; H$. hippocampus all data Mann-Whitney $U=12, N=7, P=1)$. There was no 
correlation between the density of the two seahorse species for either the data collected as ind. diver hour ${ }^{-1}$ (Spearman's rho, $r_{\mathrm{s}}=-0.1, N=5, P=0.8$ ) or in the Ria Formosa, Portugal, when abundance was measured as ind. $\mathrm{m}^{-2}$ (Spearman's rho, $r_{\mathrm{s}}=0.2, N=3, P=0.8$ ).

\section{Population structure}

Each seahorse population had its own unique combination of characteristics, regards juvenile percentage and sex ratio (Table 2). In both species, the proportion of observed juveniles varied widely, from 0 to $43 \%$ for $H$. guttulatus and from 0 to $40 \%$ for $H$. hippocampus. However, on average, this was about $17 \%$ across both species, and there appeared to be no effect of time of year. No H. guttulatus populations were significantly male-biased, but TFR2 and VBU were both significantly female-biased (Chi Squared, TFR2: $\chi^{2}$ 6.9, $P<0.01$; VBU: $\chi^{2} 8.1, P<0.01$ ), following Benjamini-Hochberg multiple comparison correction (false recovery rate 0.1 ). No $H$. hippocampus populations had a significant sex bias.

\section{Abiotic parameters of seahorse habitat}

There was a large variation in the environmental parameters of locations where seahorses were found. When all survey methods were considered, seahorses were found at depths ranging from 1 to $55 \mathrm{~m}$, but when only dive surveys were included, seahorses were just found at 1-21 m. Most $H$. guttulatus (86\%) were found at 2-5 m depth, from surveyed depths of 1-28 m. By contrast, just $19 \%$ of H. hippocampus specimens were found in $2-5 \mathrm{~m}$ depth and at two sites (GMA\&LCN) all were found much deeper $(\geq 20 \mathrm{~m})$. Neither water temperature nor visibility correlate with $H$. guttulatus abundance (Spearman's rho, water temperature $r_{\mathrm{s}}-0.03$, $N=8, P=0.96$, visibility $\left.r_{\mathrm{s}}-0.50, N=8, P=0.20\right)$, or with $H$. hippocampus abundance (Spearman's rho, water temperature $r_{\mathrm{s}}-0.38, N=6, P=0.34$, visibility $r_{\mathrm{s}} 0.04$, $N=6, P=0.94$ ) when density is calculated as ind. diver hour $^{-1}$.

\section{Seahorse habitat and holdfast preference}

New data from this study show most seahorses encountered used holdfasts, with only $1 \%$ of $\mathrm{H}$. guttulatus and $2 \%$ of $\mathrm{H}$. hippocampus seen while they were actively swimming. The seagrass Zostera marina was present at most sites (67\%) where $H$. guttulatus was observed. However seagrass beds were not the dominant habitat (6.1 SE $\pm 4.2 \%)$ in any of the sites assessed with transect dives, although it was the most popular holdfast, accounting for just under half of $\mathrm{H}$. guttulatus holdfasts (Fig. 4) (Table 1a). The second most popular holdfast type was artificial structures ( $>25 \%$ of seahorses).

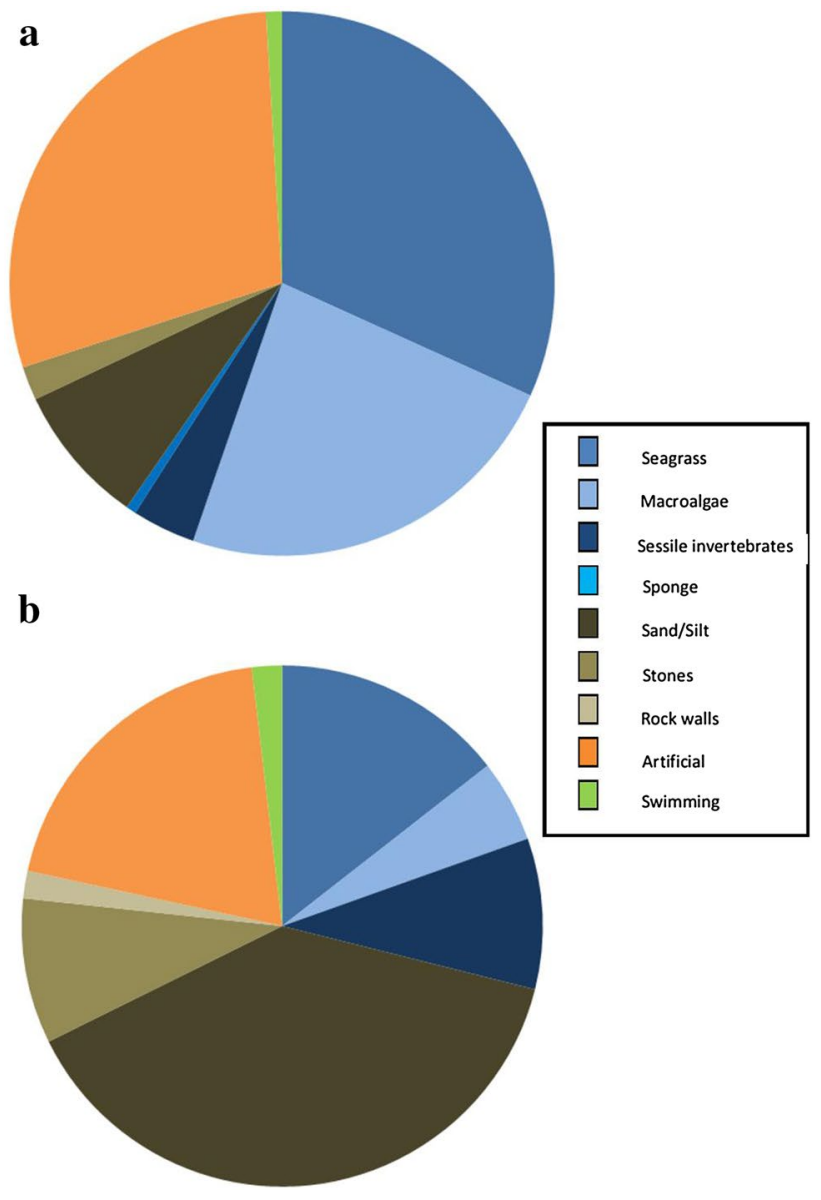

Fig. 4 Holdfast substrate types utilized by $H$. guttulatus (a) and $H$. hippocampus (b). Blues Organic, Browns Inorganic, Orange Artificial, Green No holdfast (swimming)

Artificial holdfasts were items such as tyres, fishing gear, ropes, bricks and pier supports. Transect dives revealed that most seahorses were found in complex habitats on a sand/ silt substrate. These habitats included seagrass beds, sessile invertebrates, algal species and artificial structures (Table 4).

Most sites that $H$. hippocampus inhabited were mixed habitats of open sand or silt. These substrata often had added complexity due to the presence of sessile invertebrates, artificial structures or macroalgae. In addition, at six sites at least one species of seagrass was observed in the general vicinity of the seahorses. Hippocampus hippocampus was most commonly found settled into depressions in the sediment. However artificial structures were the most common holdfast used when they were present (19\%) (Fig. 4).

Detailed descriptions of seahorse habitat and holdfast preference are lacking from many previous studies. However when reported, seagrass was present, but not dominant, at most of the sites (86\%). The seagrass observed was a mix of genera: Cymodocea, Zostera and Posidonia. However, when holdfast preference was recorded, macroalgae and artificial 
Table 4 Survey dive data, habitat types observed from transects and quadrat surveys

\begin{tabular}{|c|c|c|c|c|c|c|c|c|c|}
\hline \multirow[t]{2}{*}{ Site } & \multirow[t]{2}{*}{ Species } & \multicolumn{2}{|l|}{ Transect habitat } & \multicolumn{6}{|c|}{ Quadrat habitat and percentage cover } \\
\hline & & Dominant habitat & Other habitats & Bottom type & $\%$ cover & Flora/Fauna & $\%$ cover & Artificial & $\%$ cover \\
\hline KGR & $\begin{array}{l}\text { H. hippocampus } \\
\text { H. guttulatus }\end{array}$ & Sand & $\begin{array}{l}\text { Zostera/Cymo- } \\
\text { docea mixed } \\
\text { beds } \\
\text { Sea urchins }\end{array}$ & $\begin{array}{l}\text { Sand } \\
\text { Gravel } \\
\text { Stones } \\
\text { Rock }\end{array}$ & $\begin{array}{l}41.2 \\
38.0 \\
4.1 \\
0.2\end{array}$ & $\begin{array}{l}\text { Zostera } \\
\text { Cymodocea } \\
\text { Urchins }\end{array}$ & $\begin{array}{l}11.3 \\
3.0 \\
0.2\end{array}$ & Brick & 2.0 \\
\hline CGR & H. guttulatus & $\begin{array}{l}\text { Sand/Rock covered } \\
\text { in algae }\end{array}$ & $\begin{array}{l}\text { Gravel, urchin, } \\
\text { sea cucum- } \\
\text { ber, gravel, } \\
\text { sponge }\end{array}$ & $\begin{array}{l}\text { Sand } \\
\text { Stones } \\
\text { Rock }\end{array}$ & $\begin{array}{l}51.0 \\
33.6 \\
7.4\end{array}$ & $\begin{array}{l}\text { Zostera } \\
\text { Urchins } \\
\text { Anemone } \\
\text { Sponge }\end{array}$ & $\begin{array}{l}4.0 \\
0.8 \\
0.8 \\
1.6\end{array}$ & Brick & 0.8 \\
\hline VBU & H. guttulatus & Sand & Algae & Sand & 96.0 & $\begin{array}{l}\text { Mixed Chaetomor- } \\
\text { pha and Clad- } \\
\text { ophora spp. } \\
\text { Hermit crab }\end{array}$ & $\begin{array}{l}3.0 \\
1.0\end{array}$ & & \\
\hline $\mathrm{LCN}$ & H. hippocampus & Sand & Rock, rope, tyre & Sand & 98.0 & Rock & 0.5 & Rope tyre & $\begin{array}{l}1.0 \\
0.5\end{array}$ \\
\hline
\end{tabular}

substrates were more often used by $H$. guttulatus, and invertebrates used by $H$. hippocampus. Data were transformed into presence/absence due to the variety of methods and detail reported, but no individual factors could account for seahorse presence (ANOSIM, seagrass $R=0.26$, macroalgae $R=-0.01$, sand $R=0.09$, substrate complexity $R=0.18$ and artificial objects $R=-0.06$ ).

\section{Discussion}

The present study provides the first comprehensive review of genetic and demographic information on European seahorses throughout their geographic range. It also provides data on variability in morphology and habitat use between and within $H$. guttulatus and H. hippocampus. Despite sampling constraints, these baseline data provide useful information for conservation assessments for these data poor species of conservation concern (Woodall 2012a, b).

\section{How many species of seahorse are there in European waters?}

Genetic data from 478 samples representing 18 locations collected in 10 countries revealed the presence of five species of seahorse in NE Atlantic, Mediterranean and Black Sea waters: $H$. algiricus, $H$. erectus, $H$. fuscus, $H$. hippocampus, and $H$. guttulatus. One specimen from the Azores was identified as $H$. erectus using genetic techniques, and subsequent morphological examination has confirmed this was the first observed occurrence of this species in the eastern Atlantic Ocean (Woodall et al. 2009). Another species, H. fuscus, is an example of Lessepsian migration and was identified from specimens originating from northern Egypt, although congruence of morphological characteristics was not possible as tissue was donated rather than whole specimens. This species had previously been recorded in the south-eastern Mediterranean Sea (Golani and Fine 2002; Gokoglu et al. 2004) and extant populations have been observed as far north as Turkey (Gokoglu et al. 2004). Hippocampus algiricus, native to North-West African coasts (Czembor 2012), was observed in the Canary Islands (Otero-Ferrer et al. 2015b). By far the two most frequently seen species are $H$. guttulatus and H. hippocampus, which are native European species (Lourie et al. 2016).

There was no genetic evidence within the samples tested for the presence of cryptic species or of substantial withinspecies differentiation (i.e. of sub-species level). This conclusion could be drawn as all clades included reference sequences from known species and displayed no geographically based or morphology-related structuring, despite the high degree of intra-specific morphological variation observed within and among sites (Otero-Ferrer et al. 2017). The only substantial within-species genetic differentiation observed was that associated with haplotypes of $H$. hippocampus from Senegal and some $H$. guttulatus haplotypes from the Black Sea (Fig. 2). However in both cases no genetic and morphological correlation can be assumed, as both Black Sea and Senegalese populations also possessed the widely distributed common haplotypes of their respective species (Woodall et al. 2011, 2015). Levels of intraspecific genetic variation in cytochrome $b$ sequences for both H. guttulatus and H. hippocampus (1.23 and $1.94 \%$, respectively) were similar to values reported for other seahorse species such as $H$. barbouri (1.72\%, Lourie et al. 2005) and H. erectus $(1.44 \%$, Boehm et al. 2013). Intra-specific variation in the Control Region in H. guttulatus and H. hippocampus (1.49 and $1.96 \%$, respectively) likewise was within values given for other species such as H. abdominalis $(2.23 \%$, 
Nickel and Cursons 2012), H. capensis (1.49\%, Teske et al. 2003) and H. ingens (2.10\%, Saarman et al. 2010).

The lowest inter-specific genetic variation among seahorses has been reported between $H$. reidi and $H$. algiricus, consistent with these species having the most recent common ancestor (Teske et al. 2007a), whereas most other species pairwise comparisons show much greater genetic divergence (e.g. $5.75 \%$ between $H$. erectus and $H$. patagonicus, Boehm et al. 2013). Although not conclusive, such levels of inter-specific and intra-specific genetic variation across seahorse species suggest that the intra-specific variation observed across the entire geographical ranges of $H$. guttulatus and $H$. hippocampus is consistent with these comprising single undifferentiated species, and is also congruent with the limited morphological difference seen. This is an important conclusion, as previous studies based solely on morphological data proposed new subspecies and species in the Mediterranean Sea (Kuiter 2009) and Black Sea (Lourie et al. 1999b), that have subsequently been synonymised on the basis of the genetic data (Woodall 2012a, b). This information is crucial for surveying, assessing, monitoring, and managing the two focal species of this study, but could have wider ramifications for seahorse taxonomy globally, where it is common for morphological characters alone to be used to describe species. Integrated taxonomy is recommended for many species (Schlick-Steiner et al. 2010; Chen et al. 2011) and conclusions from this study suggest this is particularly important for seahorses where morphological differentiation can be challenging.

\section{Morphology in $H$. hippocampus and $H$. guttulatus is not consistent across their range or within populations}

Previous studies have relied on a subset of morphological characters that are distinctive in seahorses (Lourie et al. 1999b) to determine taxonomy. Data on two commonly used characteristics (presence/absence of cirri, standard length) show a differing proportion of individuals with cirri in each population of both species, and results indicate that the presence and number of cirri are unreliable characters for European seahorse species identification. This result is congruent with the few other studies that recorded this morphological character (Curtis and Vincent 2006; Curtis 2006; Louisy 2011; Tiralongo and Baldacconi 2014; Otero-Ferrer et al. 2015a). Cirri presence was more likely on larger $H$. guttulatus supporting findings by Curtis (2006), however this is not always the case (Garrick-Maidment pers. comm.), suggesting that the conditions required to exhibit this character are highly complex. In H. hippocampus however, females were more likely to have cirri. This was congruent with a study in the Canary Islands by Otero-Ferrer et al. (2015a) which reported females were more likely to have cirri than males.
Tagged H. hippocampus can shed cirri over time (JMR Curtis, unpublished data) and in some locations in the UK, $H$. hippocampus are never seen with cirri (Garrick-Maidment, unpublished data).The general shape of cirri on $H$. guttulatus and $H$. hippocampus is often different, with cirri branching in a different manner (for example images see Figure S1). However these differences are often unclear unless specimens are compared simultaneously. As the genetic data clearly confirm the presence of just two native European species, this current study can therefore confidently confirm cirri presence or absence is not a consistent or diagnosing feature within species, concurring with Curtis (2006).

Sexual dimorphism, in the form of a shorter standard length of males, was observed in H. guttulatus and H. hippocampus. This was previously reported for $H$. guttulatus (Curtis and Vincent 2006) and in many other seahorse species (Foster and Vincent 2004), but the current study is the first to indicate that this is consistent for European seahorses across their entire geographic range. Sexual dimorphism is generally a characteristic associated with polygamous species, rather than monogamous ones like seahorses (Emlen and Oring 1977; Jones and Avise 2001), although H. guttulatus is serially monogamous across breeding seasons (Naud et al. 2008). In seahorses the mating system is thought to be result from morphology, behaviour of mate competition, and the energy required to produce eggs and brood them (Kvarnemo and Simmons 2013).

Adult $H$. guttulatus from the Black Sea were significantly smaller than those from all other locations. This was observed for both new data (VBU) and published data (TTR-Kasapoglu and Duzgunes 2014). In addition, H. hippocampus from Senegal were larger than those from other sites. A significant size difference of seahorses from different populations has not been observed previously, however a large range of sizes have been reported for both $H$. hippocampus and $H$. guttulatus (Table 2), morphological variation has been seen across Macaronesia and W. Africa in H. hippocampus (Otero-Ferrer et al. 2017), and phenotypic plasticity is recognised in other seahorse species (Teske et al. 2007b). In some species of pipefish, which are in the same family as seahorses, lengths are significantly different between populations (e.g. Syngnathus floridae, Mobley and Jones 2009; Syngnathus typhle, Rispoli and Wilson 2008), and another pipefish species, Syngnathus abaster, appears to be morphologically divergent across different locations (Cakic et al. 2002; Veiga et al. 2009; Ben Alaya et al. 2011). Based on mtDNA data, these studies suggest the morphological differences between populations are probably linked to genetic differentiation in S. abaster, whereas ecological factors are a more likely cause for the morphological variation observed in other pipefish species as no genetic correlation is seen (Mobley and Jones 2009). In the present study the size of both focal species was different across sites. 
Additional studies are required to elucidate which locationspecific factors correlate with the observed size differences in seahorses. Despite the apparent trend for seahorses to be larger in the most northerly locations (a proxy of seasonal variation in temperatures), these findings are not significant and therefore neither European seahorse species follow Bergmann's rule. This rule states larger individuals are found in colder environments, and smaller ones in warmer ones. This nonconformity could be a sampling artefact, but may also reflect that an organism's size is influenced by a complex range of ecological and evolutionary processes (Berke et al. 2013), and seahorse survival requirements are known to be complex. It is therefore unsurprising that they show morphological variation across their geographic range as an adaptation to different local conditions, similar to that observed in their confamilial Syngnathus leptorhynchus (Wilson 2009).

\section{Population demographics}

Seahorse density was generally low, but patchy and highly variable. New abundance estimates were within values given in other studies of both species (Table 1), but few report density using ind. diver hour ${ }^{-1}$, which comprises the majority of new data in this study. No direct comparison between ind. $\mathrm{m}^{-2}$ and ind. diver hour ${ }^{-1}$ was possible. Safety considerations during diving, such as depth, water clarity, current flow rate and boat traffic limited the possible search area within known seahorse sites (Curtis and Vincent 2005; Curtis et al. 2017). All new study sites were chosen because seahorses had previously been observed at them, therefore abundance presented is artificially inflated. The choice of search method has also been shown to influence abundance recorded, which in most cases will have also inflated abundance reported (Correia et al. 2016). However, mean density for $H$. hippocampus from new data presented here are within values extrapolated from previous studies of $H$. hippocampus (Otero-Ferrer et al. 2015a). Data from both species combined (Goffredo et al. 2004) suggests that this method could be useful for surveys and comparisons with distance transect measures, should be a priority. Densities reported in previous studies are from transects or focal grids. The latter are often chosen to encompass areas of high seahorse density (e.g. Bell et al. 2003) and therefore seahorse densities from focal studies would be artificially higher compared to randomly placed transects. The seahorse densities given per area surveyed in the present study were generally similar to those previously reported in these species (e.g. Gristina et al. 2015), but greater than those reported for other seahorse species (Foster and Vincent 2004). This may be an artefact of the sampling protocol as mentioned above, a species-specific characteristic or peculiarity of sample location. Both abundance measures (ind. diver hour ${ }^{-1}$, ind. $\mathrm{m}^{-2}$ ) employed in the current study showed that across their range $H$. guttulatus abundance was greater than that of $H$. hippocampus. The abundance of $H$. guttulatus was always greater than that of $H$. hippocampus in locations where they co-occurred. Other studies have shown this pattern over limited geographic areas (Curtis and Vincent 2005; Caldwell and Vincent 2012; Gristina et al. 2015). Just one other study reports percentage abundance of co-occurring seahorse species, which revealed the same composition of species and the same most abundant species (Murugan et al. 2008). Counter to this in pipefish the species of greatest abundance appears to be related to season (Ripley and Foran 2006) and microhabitat preference (Malavasi et al. 2007).

The female-biased sex ratio of $H$. guttulatus in two of the sites, in the Black Sea and southern France (VBU in this study and TFR2 in Louisy 2011) is unexpected as serial monogamy reported for $H$. guttulatus in an ex situ trial and over 2 years in the wild (Naud et al. 2008) and over 4 years in the wild at a UK site (Garrick Maidment unpublished data) predicts an equal sex ratio. An independent study (new data in the present study) of site TFR reported an equal sex ratio, which might suggest the female bias of the Louisy (2011) may be an anomaly and additional data should be collected to investigate this further. Seasonal changes in the sex ratio have been reported for $H$. zostera (Strawn 1958), a female biased population was documented in H. abdominalis (Martin-Smith and Vincent 2005), and an equal sex ratio was observed for $H$. comes (Perante 2002), suggesting a variety of sex ratios can be observed across seahorse species. All $H$. hippocampus populations in the present study had an equal sex ratio, with most individuals found as male/female pairs (pers. obs.), although seasonal changes have been indicated in one study (Otero-Ferrer et al. 2015a). This interesting difference between species should be investigated further to determine if this phenomenon is a possible characteristic for niche partitioning in these species, especially as mating behaviour studies have not yet been conducted for $H$. hippocampus.

The number of juveniles seen in surveyed populations is fewer than adults. However, juveniles could be observed less frequently that adults due to the sampling method and regime or as a result of an ontogenetic habitat shift. Most studies of other seahorse species (reviewed in Foster and Vincent 2004), including H. guttulatus (Correia 2015; Gristina et al. 2017), also documented low proportions juveniles, however a high proportion has been found in some populations of $H$. capensis (Lockyear et al. 2006). There is a precedent for ontogeny in seahorses (H. comes, Morgan and Vincent 2007; H. whitei, Harasti et al. 2014). Further research is required to understand this aspect of behaviour in European seahorses, although has been observed in $H$. hippocampus in the Canary Islands (Otero-Ferrer et al. 2015a). This is especially important as best practice dictates that the 
effective management and conservation of species needs to address all life stages (Gerber and Heppell 2004).

\section{Can seahorse population location be predicted by environmental parameters?}

In this study, no individual environmental parameters could define the presence of seahorses, species abundance, or determine which species was present. However survey locations were not picked at random, with only locations where seahorses were already known to be present being studied, which may have limited our ability to detect environmental parameters that are unsuitable for seahorses. In order to model where seahorses may occur, it is important to identify how different locations and habitats fulfil the needs of seahorses. These factors could include environmental parameters under extreme events like storms and extended periods of heat (Cohen et al. 2017). Correlation of H. guttulatus abundance and temperature has been reported for multiple populations within the Ria Formosa (Correia 2015), although neither visibility nor temperature appeared to correlate with seahorse sightings in a UK site (The Seahorse Trust 2014).

When only new data were analysed, $H$. guttulatus was most commonly seen in complex habitats and $H$. hippocampus in simpler ones; supporting previous location-specific studies (Curtis and Vincent 2005; Canese et al. 2007; Correia 2015; Garrick-Maidment 2011; Gristina et al. 2015; Otero-Ferrer et al. 2015a). Niche partitioning was also observed in sympatric pipefish (Kendrick and Hyndes 2003; Malavasi et al. 2007 and in the pygmy seahorses $H$. denise and $H$. bargibanti Smith et al. 2012). There is inconclusive evidence of the importance of Zostera marina as a required or preferred habitat for $H$. guttulatus, as although it did not always co-occur with seahorse populations, when present it was most often used by $H$. guttulatus as a holdfast. Although $Z$. marina itself could be important for $H$. guttulatus, it is more likely to be the food availability as infauna and epifauna associated with the seagrass (Bostrom and Bonsdorff 1997) that is driving habitat preference, as is the case with pipefish (Ryer and Orth 1987). Individuals of both H. guttulatus and $H$. hippocampus were observed using artificial objects as holdfasts. The use of artificial holdfasts is seen in many seahorse species (Rosa et al. 2007; Clynick 2008; Faleiro et al. 2008), and could be an important factor in management measures as they could provide refuge for seahorse prey items or function as seahorse aggregation devices (Correia et al. 2013, 2015), but this apparent behavioural preference may be an observer artefact because they are more easily seen on this type of object.

The apparent wide range of habitats means that predicting the likelihood of these species' presence from habitat and environment parameters alone is challenging. This is an important consideration for environmental assessments that are made before potentially damaging activities (e.g. coastal construction). The requirements of such assessments differ across states, however habitat is often used as a precursor to determine which species (such as seahorses) could be at risk. Our findings suggest that this strategy would not be suitable for determining potential impacts on $H$. hippocampus and $H$. guttulatus. Furthermore, as seahorse conservation efforts are currently associated with seagrass conservation (e.g. Heritage Lottery Fund 2014), much of the variation in European seahorse habitat may be missed if seagrass beds alone are conserved, despite this habitat being important for many other species (McCloskey and Unsworth 2015).

\section{Important new insights and future research suggestions to enable appropriate conservation measures}

This study provides the first synthesis of data on habitat, population demography, morphology and genetics of the two native European seahorse species $H$. guttulatus and $H$. hippocampus from across their geographic range. We report the large variety of habitats in which these fish are found, failed to identify one simple parameter that predicts the presence or abundance of these seahorses, but note that seagrass is not always associated with either species. Data show that the morphology of specimens should be carefully considered together with genetic data, in an integrated approach, in order to assign species identifications (Feulner et al. 2007); such accurate integrated identification is vital in order to allow international legal mechanisms and international agreements such as the Convention on International Trade in Endangered Species (CITES 2015) to work effectively.

Emerging techniques such as eDNA screening could be applied to locate hitherto unknown populations, which would be valuable for understanding the distribution and ecology of these species. The differences in abundance observed between the two species suggest different conditions are required for these species to thrive, but these exact parameters are yet to be determined. Niche partitioning is expected in congeneric species, and further observations to determine any differences in prey items (Kitsos et al. 2008), morphology and behaviour would be an interesting contribution to determine how management measures differentially impact the two species.

As both $H$. guttulatus and H. hippocampus are currently classified as Data Deficient (IUCN 2015), any range-wide conservation measures should also encompass long-term monitoring so that the threat status of these iconic fish can be reassessed. Especially as population trends are unknown in many locations. Applying the precautionary principal, 
widespread threats from coastal development and nontarget fisheries, combined with the large geographic range, low genetic differentiation (Woodall et al. 2011, 2015) and small adult home ranges (Curtis and Vincent 2006; Curtis et al. 2017) of European seahorses suggests that a network of protected areas would be part of an effective scheme for seahorse in situ conservation. These protected areas should combine different habitat types and safeguard shallow waters, should be large enough to account for changing environmental conditions and be close enough to each other to ensure genetic and demographic connectivity. Although unlikely to be the panacea for seahorse conservation due to wider ranging issues such as climate change, where they are fished or habitat is directly damaged by human activities, protected areas have been shown to increase the size of the seahorses within them (Yasue et al. 2012) resulting in increased brood size, and thus increased resilience of the population.

Acknowledgements This is a contribution from Project Seahorse. We gratefully acknowledge the following volunteers, researchers and institutions for providing data, specimens and support during fieldwork. In France: L. Lévèque, diving unit of the Station Biologique de Roscoff, C. L. Milinaire, P. Moriniere, S. Auffret, X. De Montandouin, P. Louisy, J.-B. Senegas, P. LeLong, Ocearium du Crosic, Nausicaa, Aquairium La Rochelle, Oceanopolis, Insitu Oceanographique Paul Ricard, Universite Bordeaux, Spain: J.A. Rodriguez, Scubadoo, A.Martinez de Murguia, B. Moya, Aquarium de San Sebastian, Greece: F. Vilanikis, Y. Issaris, M. Salomidi, Tethys dive club, Thalassa, Malta: P. Camilleri, Aquaventure, Portugal, Parque Natural da Ria Formosa, M. Gaspar, F. Gil, M. Rufios. Aquario Vasco de Gama, Oceanario de Lisboa, Senegal: K. Lohse, Lanzarote: M. Frankin. Italy: A. Tavaglini, S. Repetto, L. Castellano, Oltremare, Acquario di Genova, Monaco: P. Gilles, Monaco Aquarium, Bulgaria: A. Seaman, T. Gallati, Gallati Divecenter, Volunteers: J. Marcus, V. Santos, D. Mason, M. Naud, T.-T. Ang and S. DeAmicis, S. Pocock, N. Dowden for support with field work and providing samples.

\section{Compliance with ethical standards}

Funding Funding for this project was provided by Chocolaterie Guylian and a Natural Environment Research Council Industrial Case studentship (NER/S/C/2005/13461) to LCW. Support for FOF was provided by the European Commission (ASSEMBLE project, Grant agreement no. 227799).

Conflict of interest The authors declare that they no conflicts of interest.

Ethical approval All applicable international, national, and institutional guidelines for the care and use of animals were followed.

Open Access This article is distributed under the terms of the Creative Commons Attribution 4.0 International License (http://creativecommons.org/licenses/by/4.0/), which permits unrestricted use, distribution, and reproduction in any medium, provided you give appropriate credit to the original author(s) and the source, provide a link to the Creative Commons license, and indicate if changes were made.

\section{References}

Başusta A, Özer EI, Girgin H, Serdar O, Başusta N (2014) Lengthweight relationship and condition factor of Hippocampus hippocampus and Hippocampus guttulatus inhabiting Eastern Black Sea. Pakistan J Zool 46:447-450

Bell EM, Lockyear JF, MacPherson JM, Marsden Dale, Vincent ACJ (2003) First field studies of an endangered South African seahorse, Hippocampus capensis. Environ Biol Fish 67:35-46

Ben Alaya H, Sanna D, Casu M, Biagi F, Francalacci P, Carcupino M, Galzin R, Quignard J-P, Trabelsi M (2011) Analysis of meristic and mitochondrial DNA variation in Syngnathus abaster (Teleostea: Syngnathidae) from two western Mediterranean lagoons. Biologia 66:1140-1147. https://doi.org/10.2478/ s11756-011-0127-x

Ben Amor MM, Ben Salem M, Reynaud C, Capapé C (2011) Lengthweight relationships in syngnathid species from Tunisian waters (central Mediterranean). Mar Biodivers Rec 4:e44. https://doi. org/10.1017/S1755267211000303

Berke SK, Jablonski D, Krug AZ, Roy K, Tomasovych A (2013) Beyond Bergmann's rule: size-latitude relationships in marine Bivalvia world-wide. Glob Ecol Biogeogr 22:173-183. https:// doi.org/10.1111/j.1466-8238.2012.00775.x

Boehm JT, Woodall L, Teske PR, Lourie SA, Baldwin C, Waldman J, Hickerson M, Rocha L (2013) Marine dispersal and barriers drive Atlantic seahorse diversification. J Biogeogr 40:18391849. https://doi.org/10.1111/jbi.12127

Bonaca MO, Lipej L (2005) Factors affecting habitat occupancy of fish assemblage in the Gulf of Trieste (Northern Adriatic Sea). Mar Ecol 26:42-53. https://doi. org/10.1111/j.1439-0485.2005.00037.x

Bostrom C, Bonsdorff E (1997) Community structure and spatial variation of benthic invertebrates associated with Zostera marina $(\mathrm{L}$. beds in the northern Baltic Sea. J Sea Res 37:153-166. https:// doi.org/10.1016/S1385-1101(96)00007-X

Cakic P, Lenhardt M, Mickovic D, Sekulic N, Budakov LJ (2002) Biometric analysis of Syngnathus abaster populations. J Fish Biol 60:1562-1569. https://doi.org/10.1111/j.1095-8649.2002.tb02448.x

Caldwell IR, Vincent ACJ (2012) Revisiting two sympatric European seahorse species: apparent decline in the absence of exploitation. Aquat Conserv 22:427-435. https://doi.org/10.1002/ aqc. 2238

Canese S, Giusti M, Salvati E, Angiolillo M, Cardinali A, Fabroni F, Celia-Magno M, Greci S (2007) Preliminary note of the presence and density of Hippocampus hippocampus in the Soverato Bay, Calabria Ionica. Biol Mar Mediterr 14:340-341

Casey S, Hall H, Stanley HF, Vincent AC (2004) The origin and evolution of seahorses (genus Hippocampus): a phylogenetic study using the cytochrome b gene of mitochondrial DNA. Mol Phylogenet Evol 30:261-272. https://doi.org/10.1016/j. ympev.2003.08.018

Chen J, Li Q, Kong L, Yu H (2011) How DNA barcodes complement taxonomy and explore species diversity: the case study of a poorly understood marine fauna. PLoS One 6:e21326. https:// doi.org/10.1371/journal.pone.0021326

Cisneros-Montemayor AM, West K, Boiro IS, Vincent ACJ (2016) An assessment of West African seahorses in fisheries catch and trade. J Fish Biol 88:751-759. https://doi.org/10.1111/jfb.12818

CITES (2015) What is CITES http://www.cites.org/eng.disc/what.php. Accessed 13 Nov 2015

Clynick BG (2008) Characteristics of an urban fish assemblage: distribution of fish associated with coastal marinas. Mar Environ Res 65:18-33. https://doi.org/10.1016/j.marenvres.2007.07.005

Cohen FPA, Valenti WC, Planas M, Calado R (2017) Seahorse aquaculture, biology and conservation: knowledge gaps and research 
opportunities. Rev Fish Sci Aquac 25:100-111. https://doi.org/ 10.1080/23308249.2016.1237469

Correia M (2015) Trends in seahorse abundance in the Ria Formosa, South Portugal: recent scenario and future prospects. PhD Thesis, Universidade do Algarve http://hdl.handle.net/10400.1/6822

Correia M, Palma J, Koldewey H, Andrade JP (2013) Can artificial holdfast units work as a habitat restoration tool for long-snouted seahorse (Hippocampus guttulatus Cuvier)? J Exp Mar Biol Ecol 448:258-264. https://doi.org/10.1016/j.jembe.2013.08.001

Correia M, Koldewey H, Andrade JP, Palma J (2015) Effects of artificial holdfast units on seahorse density in the Ria Formosa lagoon, Portugal. J Exp Mar Biol Ecol 471:1-7. https://doi.org/10.1016/j. jembe.2015.05.012

Correia M, Koldewey H, Andrade JP, Palma J (2016) A novel underwater visual census: seahorse population survey as a case study. Reg Stud Mar Sci 8:454-458. https://doi.org/10.1016/j. rsma.2015.10.003

Curtis JMR (2004) Life history, ecology and conservation of European seahorses. PhD Thesis, McGill University

Curtis JMR (2006) A case of mistaken identity: skin filaments are unreliable for identifying Hippocampus guttulatus and Hippocampus hippocampus. J Fish Biol 69:1855-1859. https:// doi.org/10.1111/j.1095-8649.2006.01228.x

Curtis JMR, Vincent ACJ (2005) Distribution of sympatric seahorse species along a gradient of habitat complexity in a seagrass dominated community. Mar Ecol Prog Ser 291:81-91

Curtis JMR, Vincent ACJ (2006) Life history of an unusual marine fish: survival, growth and movement patterns of Hippocampus guttulatus Cuvier 1829. J Fish Biol 68:707-733. https://doi. org/10.1111/j.0022-1112.2006.00952.x

Curtis JMR, Ribeiro J, Erzini K, Vincent ACJ (2007) A conservation trade-off? Interspecific differences in seahorse responses to experimental changes in fishing effort. Aquat Conserv 17:468-484. https://doi.org/10.1002/aqc.798

Curtis JMR, Santos SV, Nadeau JL, Gunn B, Bigney Wilner K, Balasubramanian $\mathrm{H}$, Overington S, Lesage C-M, d'Entremont J, Wieckowski K (2017) Life history and ecology of the elusive European short-snouted seahorse, Hippocampus hippocampus. J Fish Biol. https://doi.org/10.1111/jfb.13473

Czembor CA (2012) Hippocampus algiricus. The IUCN Red List of Threatened Species 2012 e.T41007A2950643 https://doi. org/10.2305/IUCN.UK.2012.RLTS.T41007A2950643.en. Accessed 17 July 2017

Dawson TP, Jackson ST, House JI, Prentice IC, Mace GM (2011) Beyond predictions: biodiversity conservation in a changing climate. Science 332:53-58. https://doi.org/10.1126/ science. 1200303

Emlen ST, Oring LW (1977) Ecology sexual selection and the evolution of mating systems. Science 197:215-223

Eschmeyer W, Fricke R (2016) Catalog of fishes: genera, species, references. http://www.calacademy.org/scientists/projects/ catalog-of-fishes. Accessed 20 July 2016

Faleiro F, Narciso L, Vicente L (2008) Seahorse behaviour and aquaculture: how to improve Hippocampus guttulatus husbandry and reproduction? Aquaculture 282:33-40. https://doi. org/10.1016/j.aquaculture.2008.05.038

Feulner PG, Kirschbaum F, Mamonekene V, Ketmaier V, Tiedemann R (2007) Adaptive radiation in African weakly electric fish (Teleostei: Mormyridae: Campylomormyrus): a combined molecular and morphological approach. J Evol Biol 20:403414. https://doi.org/10.1111/j.1420-9101.2006.01181.x

Filiz H, Taskavak E (2012) Field surveys on recent situation of seahorses in Turkey. Biharean Biol 6:55-60

Foster SJ, Vincent ACJ (2004) Life history and ecology of seahorses: implications for conservation and management. J Fish Biol 65:1-61. https://doi.org/10.1111/j.0022-1112.2004.00429.x
Franco A, Franzoi P, Malavasi S, Riccato F, Torricelli P (2006) Fish assemblages in different shallow water habitats of the Venice Lagoon. Hydrobiologia 555:159-174. https://doi.org/10.1007/ s10750-005-1113-5

Garrick-Maidment N (2011) British Seahorse Survey 2011 http:// www.theseahorsetrust.org/userfiles/PDF/BSS\%20Report\%20 2011.pdf

Garrick-Maidment N, Trewhella S, Hatcher J, Collins KJ, Mallinson JJ (2010) Seahorse tagging project, Studland Bay, Dorset, UK. Mar Biodivers Rec 3:e73. https://doi.org/10.1017/ S175526721000062X

Gerber LR, Heppell SS (2004) The use of demographic sensitivity analysis in marine species conservation planning. Biol Conserv 120:121-128. https://doi.org/10.1016/j.biocon.2004.01.029

Goffredo S, Piccinetti C, Zaccanti F (2004) Volunteers in marine conservation monitoring: a study of the distribution of seahorses carried out in collaboration with recreational scuba divers. Conserv Biol 18:1492-1503. https://doi. org/10.1111/j.1523-1739.2004.00015.x

Gokoglu M, Bodur T, Kaya Y (2004) First records of Hippocampus fuscus and Syngnathus rostellatus (Osteichthyes: Syngnathidae) from the Anatolian coast (Mediterranean Sea). J Mar Biol Assoc UK 84:1093-1094. https://doi.org/10.1017/ S0025315404010513h

Golani D, Fine M (2002) On the occurrence of Hippocampus fuscus in the eastern Mediterranean. J Fish Biol 60:764-766. https://doi. org/10.1111/j.1095-8649.2002.tb01700.x

Grima D (2011) Etat des connaissances et ressources d'informations sur les hippocampes du Bassin d'Arcachon. Extrait du rapport remis à la mission pour le parc marin du bassin d'Arcachon et son ouvert. http://www.peaubleue.org/data_folio/data_kcfinder/ files/Grima2011HippoArcachonbd.pdf

Gristina M, Cardone F, Carlucci R, Castellano L, Passarelli S, Corriero G (2015) Abundance, distribution and habitat preference of Hippocampus guttulatus and Hippocampus hippocampus in a semi-enclosed central Mediterranean marine area. Mar Ecol 36:57-66. https://doi.org/10.1111/maec.12116

Gristina M, Cardone F, Desiderato A, Mucciolo S, Lazic T, Corriero $\mathrm{G}$ (2017) Habitat use in juvenile and adult life stages of the sedentary fish Hippocampus guttulatus. Hydrobiologia 784:9-19. https://doi.org/10.1007/s10750-016-2818-3

Gurkan S, Taskavak E (2007) Length-weight relationships for syngnathid fishes of the Aegean Sea, Turkey. Belg J Zoo 137:219-222

Harasti D, Martin-Smith K, Gladstone W (2014) Ontogenetic and sex-based differences in habitat preferences and site fidelity of White's seahorse Hippocampus whitei. J Fish Biol 85:14131428. https://doi.org/10.1111/jfb.12492

Heritage Lottery Fund (2014) Pioneering research into native seahorses receives funding boost. https://www.hlf.org.uk/about-us/mediacentre/press-releases/pioneering-research-native-seahorsesreceives-funding-boost. Accessed 30 Sept 2015

Huelsenbeck JP, Ronquist F (2001) MRBAYES: Bayesian inference of phylogenetic trees. Bioinformatics 17:754-755

Issaris Y, Katsanevakis S (2010) Nondestructive distance samping surveys for population density and abundance estimates of benthic fauna. Rapp Comm Int Mer Medit 39:548

IUCN (2015) The IUCN Red List of Threatened Species. http://www. iucnredlist.org. Accessed 13 Nov 2016

Jones AG, Avise JC (2001) Mating systems and sexual selection in male-pregnant pipefishes and seahorses: insights from microsatellite-based studies of maternity. J Hered 92:150-158. https:// doi.org/10.1093/jhered/92.2.150

Kasapoglu N, Duzgunes E (2014) Some population characteristics of long-snouted seahorse (Hippocampus guttulatus Cuvier, 1829) (Actinopterygii: Syngnathidae) in the Southeastern Black Sea. Acta Zoo Bulg 66:127-131 
Kendrick AJ, Hyndes GA (2003) Patterns in the abundance and sizedistribution of syngnathid fishes among habitats in a seagrassdominated marine environment. Estuar Coast Shelf Sci 57:631640. https://doi.org/10.1016/S0272-7714(02)00402-X

Keskin C (2007) Temporal variation of fish assemblages in different shallow-water habitats in Erdek Bay, Marmara Sea, Turkey. J Black Sea/Mediterr Environ 13:215-234

Kitsos MS, Tzomos T, Anagnostopoulou L, Koukouras A (2008) Diet composition of the seahorses, Hippocampus guttulatus Cuvier, 1829 and Hippocampus hippocampus (L., 1758) (Teleostei, Syngnathidae) in the Aegean Sea. J Fish Biol 72:1259-1267. https:// doi.org/10.1111/j.1095-8649.2007.01789.x

Klein CJ, Tulloch VJ, Halpern BS, Selkoe KA, Watts ME, Steinback C, Scholz A, Possingham HP (2013) Tradeoffs in marine reserve design: habitat condition, representation, and socioeconomic costs. Conserv Lett 6:324-332. https://doi.org/10.1111/ conl.12005

Kuiter R (2009) Seahorses and their relatives. TMC Publishing ISBN10: 0977537218

Kvarnemo C, Simmons LW (2013) Polyandry as a mediator of sexual selection before and after mating. Philos Trans R Soc B 368(1613):20120042. https://doi.org/10.1098/rstb.2012.0042

Larkin MA, Blackshields G, Brown NP, Chenna R, McGettigan PA, McWilliam H, Valetin F, Wallace IM, Wilm A, Lopez A, Thompson JD, Gibson TJ, Higgins DG (2007) Clustal W and Clustal X version 2.0. Bioinformatics 23:2947-2948. https://doi. org/10.1093/bioinformatics/btm404

Lavergne S, Mouquet N, Thuiller W, Ronce O (2010) Biodiversity and climate change: integrating evolutionary and ecological responses of species and communities. Annu Rev Ecol Evol S 41:321-350. https://doi.org/10.1146/annurev-ecolsys-102209-144628

Lockyear JF, Hecht T, Kaiser H, Teske PR (2006) The distribution and abundance of the endangered Knysna seahorse Hippocampus capensis (Pisces: Syngnathidae) in South African estuaries. Afr J Aquat Sci 31:275-283. https://doi. org/10.2989/16085910609503897

Louisy A (2011) Hippo-THAU Bilan Scientifique 2005-2009. Association Peau-Bleue

Lourie SA, Vincent AC, Hall H (1999a) Seahorses: an identification guide to the world's species and the conservation. Project Seahorse and TRAFFIC North America, Washington

Lourie S, Pritchard J, Casey S, Truong S, Hall H, Vincent A (1999b) Taxonomy of Vietnam's exploited seahorses (family Syngnathidae). Biol J Linn Soc 66:231-256. https://doi. org/10.1111/j.1095-8312.1999.tb01886.x

Lourie SA, Green DM, Vincent AC (2005) Dispersal, habitat differences, and comparative phylogeography of Southeast Asian seahorses (Syngnathidae: Hippocampus). Mol Ecol 14:1073-1094. https://doi.org/10.1111/j.1365-294X.2005.02464.x

Lourie SA, Pollom RA, Foster SJ (2016) A global revision of the seahorses Hippocampus Rafinesque 1810 (Actinopterygii: Syngnathiformes): taxonomy and biogeography with recommendations for further research. Zootaxa 4146:1-66. https://doi. org/10.11646/zootaxa.4146.1.1

Malavasi S, Franco A, Riccato F, Valerio C, Torricelli P, Franzoi P (2007) Habitat selection and spatial segregation in three pipefish species. Estuar Coast Shelf Sci 75:143-150. https://doi. org/10.1016/j.ecss.2007.02.022

Martin-Smith KM, Vincent ACJ (2005) Seahorse declines in the Derwent estuary, Tasmania in the absence of fishing pressure. Biol Conserv 123:533-545. https://doi.org/10.1016/j. biocon.2005.01.003

McCloskey RM, Unsworth RKF (2015) Decreasing seagrass density negatively influences associated fauna. PeerJ 3:e1053. https:// doi.org/10.7717/peerj.1053
Mobley KB, Jones AG (2009) Environmental, demographic, and genetic mating system variation among five geographically distinct dusky pipefish (Syngnathus floridae) populations. Mol Ecol 18:14761490. https://doi.org/10.1111/j.1365-294X.2009.04104.x

Morgan SK, Vincent ACJ (2007) The ontogeny of habitat associations in the tropical tiger tail seahorse Hippocampus comes Cantor, 1850. J Fish Biol 71:701-724. https://doi. org/10.1111/j.1095-8649.2007.01535.x

Murugan A, Dhanya S, Rajagopal S, Balasubramamian T (2008) Seahorses and pipefishes of the Tamil Nadu coast. Curr Sci India 95:253-260

Nadeau JL, Curtis JMR, Lourie SA (2009) Preservation causes shrinkage in seahorses: implications for biological studies and for managing sustainable trade with minimum size limits. Aquat Con 19:428-438. https://doi.org/10.1002/aqc.1002

JNCC, Natural England (2012) Marine Conservation Zone Project: JNCC and Natural England's advice to DEFRA on recommended Marine Conservation Zones

Naud MJ, Curtis JMR, Woodall LC, Gaspar MB (2008) Mate choice, operational sex ratio, and social promiscuity in a wild population of the long-snouted seahorse Hippocampus guttulatus. Behav Ecol 20:160-164. https://doi.org/10.1093/beheco/arn128

Nickel J, Cursons R (2012) Genetic diversity and population structure of the pot-belly seahorse Hippocampus abdominalis in New Zealand. N Zeal J Mar Fresh 46:207-218. https://doi.org /10.1080/00288330.2011.632014

Otero-Ferrer F (2011) Seahorses in Gran Canaria Island (Spain): ecology and aquaculture-combined tools for marine conservation issues. PhD Thesis, Universidad de Las Palmas de Gran Canaria. http://hdl.handle.net/10553/8709

Otero-Ferrer F, Herrera R, Tuset VM, Socorro J, Molina L (2015a) Spatial and seasonal patterns of European short-snouted seahorse Hippocampus hippocampus distribution in island coastal environments. Afr J Mar Sci 37:395-404. https://doi.org/10.2 989/1814232X.2015.1083476

Otero-Ferrer F, Herrera R, López A, Socorro J, Molina L, Bouza C (2015b) First records of Hippocampus algiricus in the Canary Islands (north-east Atlantic Ocean) with an observation of hybridization with Hippocampus hippocampus. J Fish Biol 87:1080-1089. https://doi.org/10.1111/jfb.12760

Otero-Ferrer F, González JA, Freitas M, Araújo R, Azevedo JMN, Holt WV, Tuya F, Haroun R (2017) When natural history collections reveal secrets on data deficient threatened species: Atlantic seahorses as a case study. Biodivers Conserv. https:// doi.org/10.1007/s10531-017-1385-x

Padial JM, Miralles A, De la Riva I, Vences M (2010) The integrative future of taxonomy. Front Zool 7:16. https://doi. org/10.1186/1742-9994-7-16

Perante N (2002) Biology of a seahorse species, Hippocampus comes in the central Philippines. J Fish Biol 60:821-837. https://doi. org/10.1111/j.1095-8649.2002.tb02412.x

Perry A, Low P, Ellis J, Reynolds J (2005) Climate change and distribution shifts in marine fishes. Science 308:1912-1915. https:// doi.org/10.1126/science.1111322

Posada D, Crandall KA (2001) Selecting the best-fit model of nucleotide substitution. Syst Biol 50:580-601. https://doi. org/10.1080/10635150118469

Ripley JL, Foran CM (2006) Population structure, growth rates, and seasonal abundance of two Syngnathus pipefish species. Estuar Coast 29:1161-1171. https://doi.org/10.1007/BF02781817

Rispoli VF, Wilson AB (2008) Sexual size dimorphism predicts the frequency of multiple mating in the sex-role reversed pipefish Syngnathus typhle. J Evol Biol 21:30-38. https://doi. org/10.1111/j.1420-9101.2007.01470.x

Rosa IL, Oliveira TPR, Castro ALC, de Souza Moraes LE, Xavier JHA, Nottingham MC, Dias TLP, Bruto-Costa LV, Araújo 
ME, Birolo AB, Mai ACG, Monteiro-Neto C (2007) Population characteristics, space use and habitat associations of the seahorse Hippocampus reidi (Teleostei: Syngnathidae). Neotrop Ichthyol 5:405-414. https://doi.org/10.1590/ S1679-62252007000300020

Ryer CH, Orth RJ (1987) Feeding ecology of the Northern Pipefish, Syngnathus fuscus, in a seagrass community of the Lower Chesapeake Bay. Estuar 10:330-336. https://doi. org/10.2307/1351891

Saarman NP, Louie KD, Hamilton H (2010) Genetic differentiation across eastern Pacific oceanographic barriers in the threatened seahorse Hippocampus ingens. Conserv Genet 11:1989-2000. https://doi.org/10.1007/s10592-010-0092-x

Schlick-Steiner B, Steiner FM, Sifert B, Stauffer C, Christian E, Crozier RH (2010) Integrative taxonomy: a multisource approach to exploring biodiversity. Annu Rev Entomol 55:421-438. https:// doi.org/10.1146/annurev-ento-112408-085432

Schmitt EF, Sullivan KM (1996) Analysis of a volunteer method for collecting fish presence and abundance data in the Florida Keys. Bull Mar Sci 59:404-416

Selig ER, Turner WR, Troëng S, Wallace BP, Halpern BS, Kaschner K, Lascelles BG, Carpenter KE, Mittermeier RA (2014) Global priorities for marine biodiversity conservation. PLoS One 9:e82898. https://doi.org/10.1371/journal.pone.0082898

Smith RE, Grutter AS, Tibbetts IR (2012) Extreme habitat specialisation and population structure of two gorgonian-associated pygmy seahorses. Mar Ecol Prog Ser 444:195-206. https://doi. org/10.3354/meps09471

Strawn K (1958) Life history of the pigmy seahorse, Hippocampus zosterae Jordan and Gilbert, at Cedar Key, Florida. Copedia 1:16-22

Teske PR, Cherry MI, Matthee CA (2003) Population genetics of the endangered Knysna seahorse, Hippocampus capensis. Mol Ecol 12:1703-1715. https://doi. org/10.1046/j.1365-294X.2003.01852.X

Teske PR, Hamilton H, Matthee CA, Barker NP (2007a) Signatures of seaway closures and founder dispersal in the phylogeny of a circumglobally distributed seahorse lineage. BMC Evol Biol 7:138. https://doi.org/10.1186/1471-2148-7-138

Teske PR, Lourie SA, Matthee CA, Green DM (2007b) Hippocampus queenslandicus Horne, 2001-a new seahorse species or yet another synonym? Aust J Zool 55:139-145. https://doi. org/10.1071/ZO07021

The Seahorse Trust (2014) Year 5 report on the seahorse tagging project at South Beach, Studland Bay in Dorset run by The Seahorse Trust. http://www.theseahorsetrust.org/userfiles/Year\%205\%20 report $\% 20$ on $\% 20$ the $\% 20$ Tagging $\% 20$ of\%20Seahorses $\% 20$ at $\% 20$ Studland\%20Bay\%20i\%20Dorset.pdf

Tiralongo F, Baldacconi R (2014) A conspicuous population of the long-snouted seahorse, Hippocampus guttulatus (Actinopterygii: Syngnathiformes: Syngnathidae), in a highly polluted Mediterranean coastal lagoon. Acta Ichthyol Piscat 44:99-104

Valladares S, Quintas P, Blanco A, Chamorro A, Hermelo T, Planas M (2011) Description of Hipocampus guttulatus populations in Punta Cabalo (Ria de Arousa, NW Spain). In: 1st Syngnathid Biology Symposium, Fiskebackskil, Sweden

Valladares S, Banon R, Lopez A, Bouza C, Chamorro A, Garcia ME, Planas M (2013) First records of the seahorse Hippocampus hippocampus in Galician waters (NW Spain). Cybium 38:74-76
Vasil'Eva E (2007) Seahorse species (genus Hippocampus, Pisces) described by C. Linné. Folia Zool 56:319-327

Veiga P, Machado D, Almeida C, Bentes L, Monteiro P, Oliveira F, Ruano M, Erzini K, Gonçalves JMS (2009) Weightlength relationships for 54 species of the Arade estuary, southern Portugal. J Appl Ichthyol 25:493-496. https://doi. org/10.1111/j.1439-0426.2009.01230.x

Verdiell-Cubedo D, Oliva-Paterna FJ, Torralva M (2006) Lengthweight relationships for 22 fish species of the Mar Menor coastal lagoon (western Mediterranean Sea). J Appl Ichthyol 22:293294. https://doi.org/10.1111/j.1439-0426.2006.00738.x

Verdiell-Cubedo J, Oliva-Paterna FJ, Egea-Serrano A, Torralva-Forero M (2008) Population biology and habitat associations of benthic fish species in the shallow areas of a Mediterranean coastal lagoon (SE Iberian Peninsula). Sci Mar 72:319-328. https://doi. org/10.3989/scimar.2008.72n2319

Vieira R, Monteiro P, Ribeiro J, Bentes L, Oliveira F, Erzini K, Goncalves J (2014) Length-weight relationships of six syngnathid species from Ria Formosa, SW Iberian coast. Cah Biol Mar $55: 9-12$

West K (2012) Investigations into the Senegalese trade in CITES-listed seahorses, Hippocampus algiricus. MSc Thesis, Imperial College London

Wilson AB (2009) Fecundity selection predicts Bergmann's rule in syngnathid fishes. Mol Ecol 18:1263-1272. https://doi. org/10.1111/j.1365-294X.2009.04084.x

Woodall LC (2012a) Hippocampus guttulatus. The IUCN Red List of Threatened Species 2012 e. T41006A16997706. https://doi. org/10.2305/IUCN.UK.2012.RLTS.T41006A16997706.en. Accessed 10 July 2017

Woodall LC (2012b) Hippocampus hippocampus. The IUCN Red List of Threatened Species 2012 e.T10069A17338787 https:// doi.org/10.2305/IUCN.UK.2012.RLTS.T10069A17338787.en. Accessed 10 July 2017

Woodall LC, Koldewey HJ, Santos SV, Shaw PW (2009) First occurrence of the lined seahorse Hippocampus erectus in the eastern Atlantic Ocean. J Fish Biol 75:1505-1512. https://doi. org/10.1111/j.1095-8649.2009.02371.x

Woodall LC, Koldewey HJ, Shaw PW (2011) Historical and contemporary population genetic connectivity of the European shortsnouted seahorse Hippocampus hippocampus and implications for management. J Fish Biol 78:1738-1756. https://doi. org/10.1111/j.1095-8649.2011.02974.x

Woodall LC, Koldewey HJ, Boehm JT, Shaw PW (2015) Past and present drivers of population structure in a small coastal fish, the European long snouted seahorse Hippocampus guttulatus. Conserv Genet 16:1139-1153. https://doi.org/10.1007/ s10592-015-0728-y

Yasue M, Nellas A, Vincent ACJ (2012) Seahorses helped drive creation of marine protected areas, so what did these protected areas do for the seahorses? Environ Conserv 39:183-193. https://doi. org/10.1017/S0376892911000622 